\title{
Genome of the pincer wasp Gonatopus flavifemur reveals unique venom evolution and a dual adaptation to parasitism and predation
}

Yi Yang ${ }^{1+} \mathbb{D}$, Xinhai Y $e^{1+} \mathbb{D}$, Cong Dang ${ }^{1}$, Yunshen Cao ${ }^{1}$, Rui Hong ${ }^{1}$, Yu H. Sun ${ }^{2}$, Shan Xiao ${ }^{1}$, Yang Mei $^{1}$, Le Xu', Qi Fang ${ }^{1}$, Huamei Xiao ${ }^{1,3}$, Fei $\mathrm{Li}^{1}$ (1) and Gongyin Ye ${ }^{1 *}$ (i)

\begin{abstract}
Background: Hymenoptera comprise extremely diverse insect species with extensive variation in their life histories. The Dryinidae, a family of solitary wasps of Hymenoptera, have evolved innovations that allow them to hunt using venom and a pair of chelae developed from the fore legs that can grasp prey. Dryinidae larvae are also parasitoids of Auchenorrhyncha, a group including common pests such as planthoppers and leafhoppers. Both of these traits make them effective and valuable for pest control, but little is yet known about the genetic basis of its dual adaptation to parasitism and predation.

Results: We sequenced and assembled a high-quality genome of the dryinid wasp Gonatopus flavifemur, which at $636.5 \mathrm{Mb}$ is larger than most hymenopterans. The expansion of transposable elements, especially DNA transposons, is a major contributor to the genome size enlargement. Our genome-wide screens reveal a number of positively selected genes and rapidly evolving proteins involved in energy production and motor activity, which may contribute to the predatory adaptation of dryinid wasp. We further show that three female-biased, reproductiveassociated yellow genes, in response to the prey feeding behavior, are significantly elevated in adult females, which may facilitate the egg production. Venom is a powerful weapon for dryinid wasp during parasitism and predation. We therefore analyze the transcriptomes of venom glands and describe specific expansions in venom Idgf-like genes and neprilysin-like genes. Furthermore, we find the LWS2-opsin gene is exclusively expressed in male G. flavifemur, which may contribute to partner searching and mating.

Conclusions: Our results provide new insights into the genome evolution, predatory adaptation, venom evolution, and sex-biased genes in G. flavifemur, and present genomic resources for future in-depth comparative analyses of hymenopterans that may benefit pest control.
\end{abstract}

Keywords: Parasitoid wasp, Dryinidae, Genome size, Predation, Genome sequencing, Venom

\footnotetext{
* Correspondence: chu@zju.edu.cn

${ }^{\dagger}$ Yi Yang and Xinhai Ye contributed equally to this work.

'State Key Laboratory of Rice Biology and Ministry of Agricultural and Rural

Affairs Key Laboratory of Molecular Biology of Crop Pathogens and Insects,

Zhejiang University, Hangzhou, China

Full list of author information is available at the end of the article
}

\section{$\triangle B M C$}

(c) The Author(s). 2021 Open Access This article is licensed under a Creative Commons Attribution 4.0 International License, which permits use, sharing, adaptation, distribution and reproduction in any medium or format, as long as you give appropriate credit to the original author(s) and the source, provide a link to the Creative Commons licence, and indicate if changes were made. The images or other third party material in this article are included in the article's Creative Commons licence, unless indicated otherwise in a credit line to the material. If material is not included in the article's Creative Commons licence and your intended use is not permitted by statutory regulation or exceeds the permitted use, you will need to obtain permission directly from the copyright holder. To view a copy of this licence, visit http://creativecommons.org/licenses/by/4.0/. The Creative Commons Public Domain Dedication waiver (http://creativecommons.org/publicdomain/zero/1.0/) applies to the data made available in this article, unless otherwise stated in a credit line to the data. 


\section{Background}

Hymenoptera are an extremely diverse insect order with a variety of life history traits, including phytophagy, parasitism, predation, pollination, and eusociality, providing an ideal model for studying the evolutionary origin and transition of some key traits $[1,2]$. Dryinidae are a family within Chrysidoidea, which have several intriguing biological properties [3, 4]. These wasps are both parasitoids and predators of Auchenorrhyncha hosts (e.g., planthoppers) belonging to the order Hemiptera. Female wasps lay eggs on the hosts, and their young offspring develop outside the hosts (ectoparasitioid) (Fig. 1c). Also, female wasps catch and feed on the hosts. The protein-rich diets might be beneficial for egg production [4]. However, male wasps do not hunt or feed on hosts. The parasitoid wasps with both predatory and parasitoid behaviors are rare in Hymenoptera. Additionally, no other wasps with predatory behavior are found in Chrysidoidea [5]. Thus, the origin of predatory behavior in Dryinidae is likely an independent trait gaining event in the evolution of Hymenoptera. Moreover, Dryinidae are highly sexual dimorphic. The adult females are ant-like wasps and often wingless, whereas the adult males are winged [3] (Fig. 1a, b). Particularly, the fore legs of adult females evolved to be a pair of robust chelae, which are useful in prey capturing [3, 4] (Fig. 1d, d'). Therefore, dryinid wasp is also known as pincer wasp. The female adults have evolved a mimicry of antlike body, which allows them to attack their hosts easily, as ants usually feed on the honeydew produced by Auchenorrhyncha insects [4]. Interestingly, these femalespecific features seem to be related to their predatory behavior. However, very little is known about the genetic basis and evolutionary history of Dryinidae's adaptation to their special parasitoid-predatory life.

Dryinidae provides a promising model to study the origin of predatory behavior and sexual dimorphism. Gonatopus flavifemur is a common parasitoid of the notorious rice pest, the brown planthopper Nilaparvata lugens [6-8]. Its unique predatory and parasitoid behaviors make this species very effective for the biological control of pests $[9,10]$. Here, we report the genomic resources of G. flavifemur, representing the first genome sequence of the family Dryinidae. This $636.5 \mathrm{Mb}$ genome assembly is much larger than most hymenopterans, due to the massive expansion of transposable element sequences. Analysis of the G. flavifemur genome highlights several positively selected genes and rapidly evolving proteins likely involved in major aspects of predatory adaptation. Gene expression changes in female adults

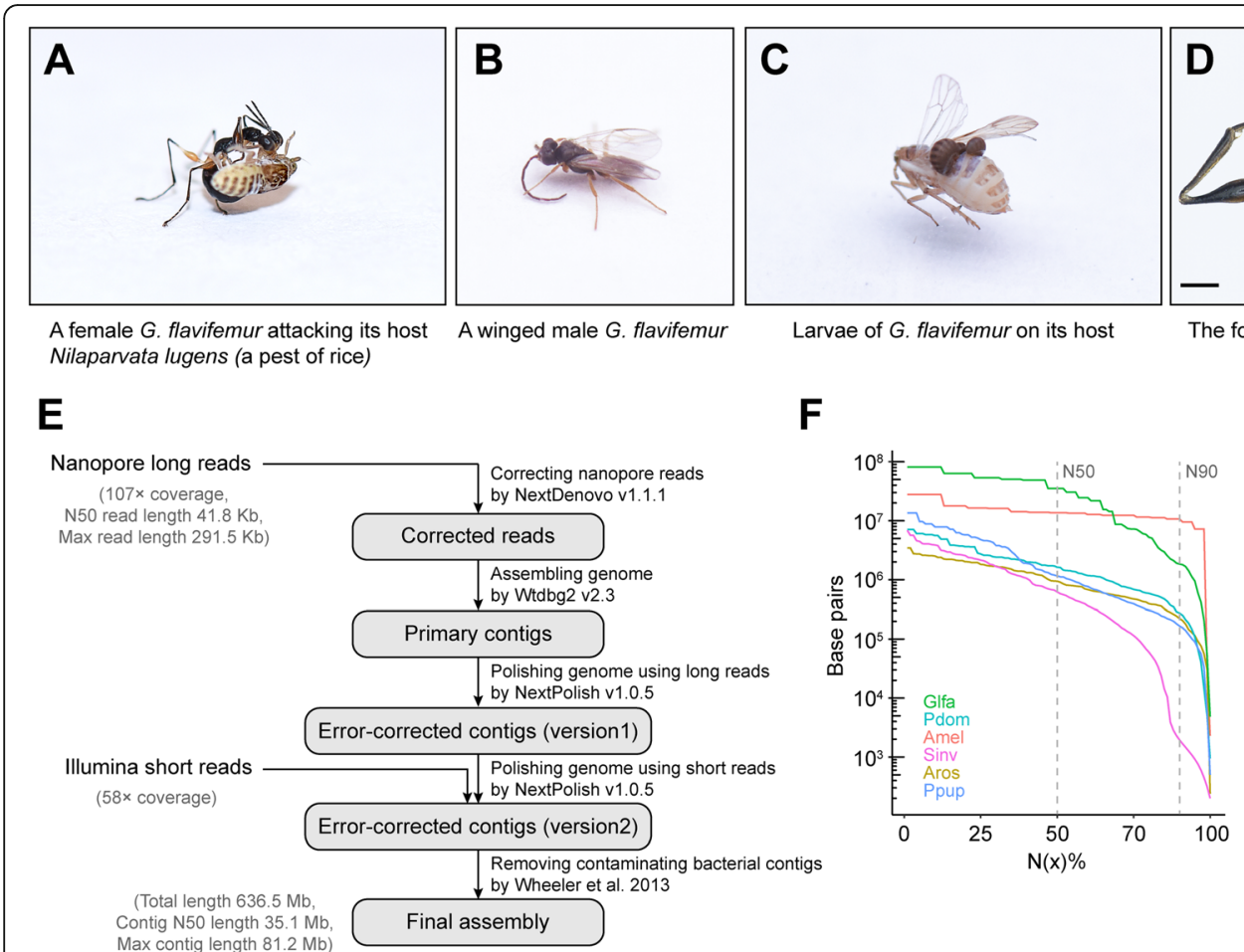

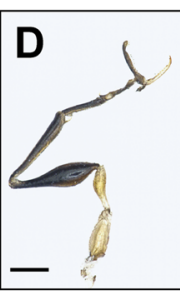

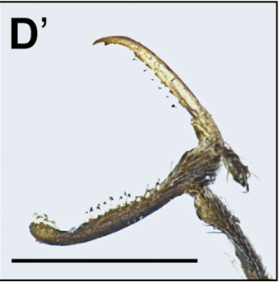

he fore leg and chela of female G. flavifemur

\section{G}

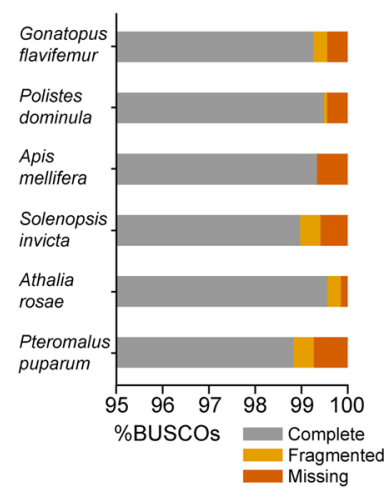

Fig. 1 Assembly of the genome of G. flavifemur. a A female G. flavifemur attacking its host, the brown planthopper Nilaparvata lugens. b A winged male G. flavifemur. c Larvae of G. flavifemur on its host. d, d' The fore leg and chela of female G. flavifemur. Scale bars: 300 $\mu m$. e An overview of the genome assembly strategy. $\mathbf{f}$ Comparison of assembly contiguity among six hymenopterans. $\mathrm{N}(\mathrm{x}) \%$ graphs show contig or scaffold sizes (y-axis), in which $x$ percent of the assembly consists of contigs/scaffolds of at least that size. $\mathbf{g}$ Comparison of the completeness of genome assemblies, as a percentage of 1367 insect genes from insecta_odb10 
after feeding on the preys are featured. Due to the importance of venom to the wasps, we also provide insights into the venom-associated genes, and describe specific expansions in venom Idgf-like genes and neprilysin-like genes. Finally, we identify many sex-biased genes which may be related to sexual dimorphism. In sum, our findings provide insights into the genome size evolution, parasitoid-predatory adaptation, venom evolution, and sexual dimorphism. In addition, this genome underpins further research of G. flavifemur and greatly facilitates future analyses of the trait evolution in Hymenoptera.

\section{Results}

\section{Sequencing, assembly, and annotation}

We generated Nanopore long reads (107X genome coverage) and Illumina short reads (58X genome coverage) from 50 male pupae for genome assembly (Fig. 1e; Additional file 1: Supplementary Table $1-4$ ). The genome sizes of G. flavifemur estimated by flow cytometry and K-mer analysis were about 601.4 and $603.4 \mathrm{Mb}$, respectively (Additional file 1: Supplementary Table 5, Additional file 2: Supplementary Figure $1-2$ ). After filtering out the bacterial contaminating contigs $(1.7 \mathrm{Mb})$ (see Additional file 3 for details about the removal of bacterial contaminating contigs) [11-26], we obtained a $636.5 \mathrm{Mb}$ high-contiguity genome assembly of G. flavifemur, with a contig N50 of $35.1 \mathrm{Mb}$. The maximum contig length reaches $81.2 \mathrm{Mb}$ (Fig. 1e). We compared this genome assembly to other five high-quality genome assemblies in Hymenoptera, showing that our assembly has a higher contig N50 value (Fig. 1f). Although Hi-C or other technologies were not applied to improve our assembly to super-scaffold level, we found that the contig N50 value of our assembly is higher than the scaffold N50 values of two chromosome-level genomes in $\mathrm{Hy}-$ menoptera (Apis mellifera and Pteromalus puparum) $[13,27]$. Such very long contigs we obtained reflect the power of Nanopore long-read sequencing technology and related long-read-aware strategies in assembling a high-contiguity genome. Additionally, the appearance of very long contigs might be due to the larger genome size of G. flavifemur, which is 2.8 times the length of $A$. mellifera $(255 \mathrm{Mb})$ and 1.9 times the length of $P$. puparum (338 Mb). Moreover, the haploid chromosome number of Gonatopus wasps is four [28]. Based on this information, the average chromosome length of G. flavifemur is $159.1 \mathrm{Mb}$, which is much larger than the maximum contig length of our assembly $(81.2 \mathrm{Mb})$. Thus, the Nanopore long-read sequencing and assembly strategy, the large genome size, and low chromosome number explain the very long contigs obtained in our assembly.

Genome assessment using Benchmarking Universal Single-Copy Orthologs (BUSCO) indicated $99.3 \%$ of the insect gene set are present and complete (Fig. 1g). This
BUSCO score is similar to other high-quality genomes. In addition, our analyses showed that $97.84 \%$ of Illumina whole-genome sequencing reads and $95.60 \%$ of RNA-seq reads could be properly mapped to the genome assembly respectively (Additional file 1: Supplementary Table 6). Thus, both BUSCO result and mapping quality indicated that our genome assembly is highly accurate and nearly complete. We annotated protein-coding genes by combining the evidence from homology alignments, de novo predictions and gene expressions. Totally, 23,100 protein-coding genes were identified in the genome. Comparing to a total number of 24,388 genes predicted in Nasonia vitripennis, our annotation is similar, but slightly larger than other hymenopterans. Moreover, we further identified several gene families that are important and popular in insect studies, including 66 cytochrome P450s, 17 glutathione S-transferases (GSTs), 35 ATP-binding cassette transporters (ABCs), 10 gustatory receptors (GRs), 20 ionotropic receptors (IRs), 43 olfactory receptors (ORs), 8 odorant binding proteins (OBPs), 8 sensory neuron membrane proteins (SNMPs), and 6 chemosensory proteins (CSPs) (Additional file 1: Supplementary Table 7). The gene numbers of detoxificationrelated gene families (P450, GST, ABC) in G. flavifemur are comparable to other hymenopteran insects we tested. However, in contrast to the detoxification genes, G. flavifemur has fewer chemosensory genes (OR, GR, OBP) than other hymenopteran insects, which may be explained by its relatively narrow host range (the main host is the brown planthopper, $N$. lugens) and unitary living environment (rice fields).

\section{Phylogenomics}

We chose 13 representative hymenopteran insects (including G. flavifemur) for phylogenomic analyses and the following comparative genomics analyses because of their high genome qualities, popularities, and evolutionary positions. Our phylogenomic analyses were based on 2992 single-copy genes, which were firstly identified by OrthoFinder from the genomes of 13 hymenopterans (one sawfly, one parasitic wood wasp, two braconid wasps, three chalcid wasps, one paper wasp, two ants, two bees, and one dryinid wasp). The amino acid sequences of the single-copy gene set were aligned and concatenated, followed by a phylogenetic tree construction. In this analysis, dryinid wasp G. flavifemur (from the superfamily Chrysidoidea) was placed as the sister group to all other Aculeate members (paper wasp, ants, and bees) after their common ancestor diverged from the infraorder Parasitoida (Fig. 2a, Additional file 2: Supplementary Figure 3a). In addition, we conducted coalescent-based analyses using ASTRAL by considering gene trees from the single-copy gene set individually, and obtained the same topology (Additional file 2: 

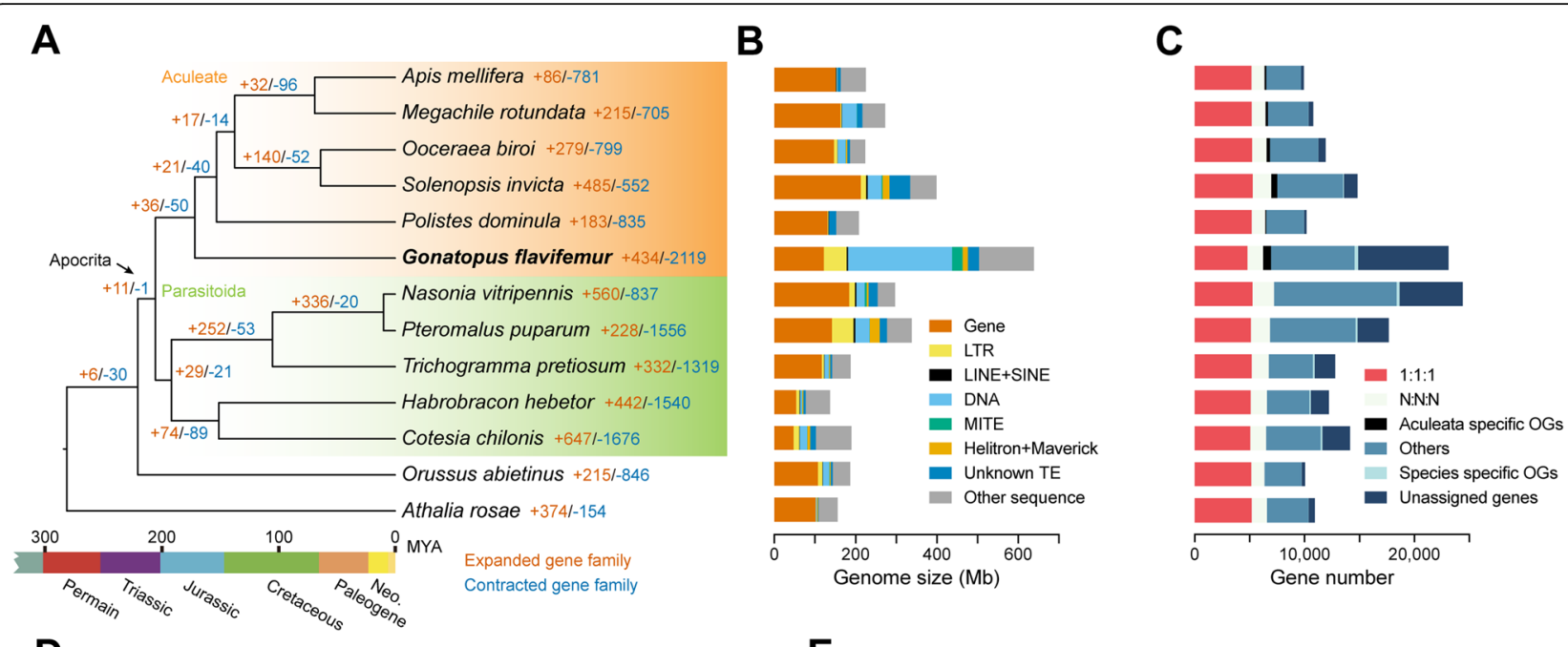

D

E
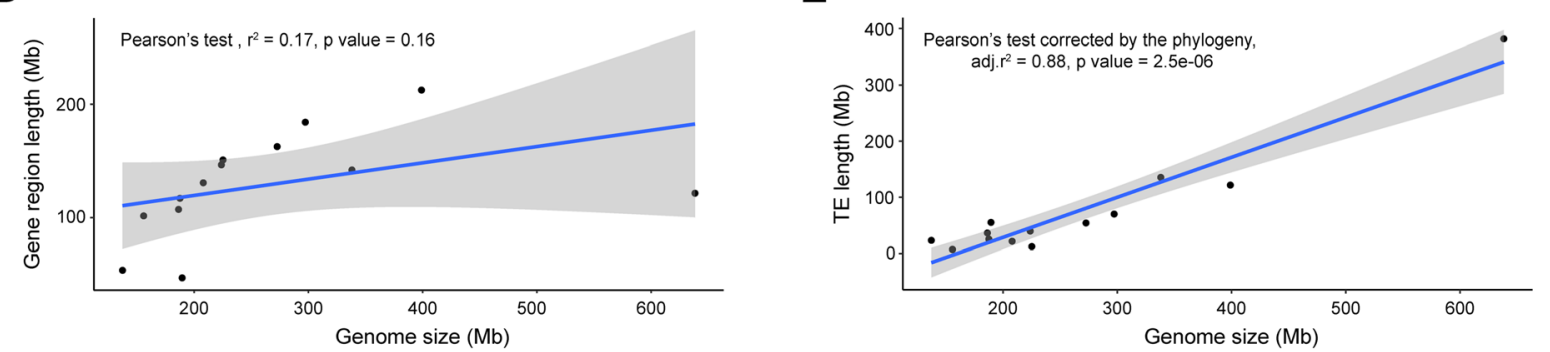

Fig. 2 Phylogenetic and comparative genomic analysis of G. flavifemur. a The maximum likelihood phylogenetic tree built from 2992 concatenated single-copy orthologous groups from G. flavifemur and other 12 hymenopterans using IQ-TREE. The basal hymenopteran A. rosae was used as an outgroup. All nodes received 100\% bootstrap support. The expansion numbers of gene families (orange) and contraction (blue) are shown on the branches. $\mathbf{b}$ Bar plots show total number of nucleotides occupied by genomic components. $\mathbf{c}$ Total gene counts of different types of orthologous groups in each genome. "1:1:1" indicates universal single-copy genes present in all species, absence and/or duplicated in, at most, one genome is included; "N:N:N" indicates other universal genes; "Aculeata specific OGs" indicates common unique genes in the six Aculeata species. "Species-specific OGs" represents species-specific genes with more than one copy in the genome. "Unsigned genes" indicates species-specific genes with only one copy in the genome. "Others" indicates the remaining genes. $\mathbf{d}$, e The contribution of coding DNA sequence and TEs to genome size evolution across Hymenoptera. Lines correspond to linear regressions; shadows correspond to the 95\% confidence intervals around the mean predictions

Supplementary Figure 3B). G. flavifemur diverged from other aculeates approximately 171.6 million years ago, during the Jurassic period (Fig. 2a). Our results indicate that the superfamily Chrysidoidea (including dryinid wasp) represents an early branch of the Aculeate, and this phylogenetic position is also supported by previous studies using transcriptome data and ultra-conserved elements $[1,2]$. Filling the gap of the genomic sequences of this key phylogenetic position will greatly facilitate future comparative studies in Hymenoptera evolution.

\section{Genome size and transposable element}

The hymenopteran genomes are moderate in size $(80 \%$ are between 180 and $340 \mathrm{Mb}$ ) based on current sequencing projects [29]. However, there are a few exceptions [30], for example, the orchid bee Euglossa dilemma (3.3 $\mathrm{Gb})$ [31] and the gall wasp Belonocnema treatae (1.5 Gb) (NCBI RefSeq assembly accession: GCF_010883055.1).
The genome size of dryinid wasp G. flavifemur is 636.5 $\mathrm{Mb}$, representing a relatively large genome in Hymenoptera. The expansion of repetitive sequences (e.g., transposable elements, TEs) is one of the most important factors to enlarge the genome size, and this phenomenon has been reported in many insects [30, 32-34]. Unsurprisingly, G. flavifemur genome contains massive repeat sequences, comprising approximately $60.7 \%$ of the whole genome. TEs account for around $59.9 \%(381.7 \mathrm{Mb})$ of the G. flavifemur genome, with DNA transposons being the most abundant TE group $(40.3 \%$; $256.3 \mathrm{Mb})$ (Fig. 2b). Based on the phylogeny of Hymenoptera, we noticed that TE content is strongly correlated with genome size (Fig. 2e; adj. $\mathrm{r}^{2}=0.88, p=$ $2.5 \mathrm{e}-06$, Pearson's test corrected by the phylogeny), while gene region length shows weak correlation with genome size (Fig. 2d; adj. $\mathrm{r}^{2}=0.17, p=0.16$, Pearson's test). This result suggested that TE is a strong factor to 
drive genome size enlargement in the evolution of $\mathrm{Hy}$ menoptera. This pattern is supported by a previous study focused on aculeates [35]. The basal hymenopteran insect Athalia rosae $(155.8 \mathrm{Mb})$ has a moderate genome size and a low TE content (4.6\%), which may imply the ancestral state of hymenopteran genome. During the evolution of Hymenoptera, TE expansion likely happened independently after species divergence, resulting in genome size enlargement. In addition to the TE expansion of G. flavifemur, we found another obvious example, $P$. puparum, which also has abundant expanded TEs (about 40.1\%) in its genome [13]. To figure out the contribution of each TE class to the genome size enlargement in Hymenoptera, we compared genome size differences with the content differences of each type of TE. The results showed that the expansion of DNA transposons to a large extent contributes to the genome size enlargement in Hymenoptera (Fig. 3a; adj. $\mathrm{r}^{2}=0.77$, $p=8.0 \mathrm{e}-05$, Pearson's test corrected by the phylogeny). We also detected moderate positive correlations between the content of long terminal repeat retrotransposons (LTRs) (adj. $\mathrm{r}^{2}=0.35, p=0.035$, Pearson's test corrected by the phylogeny), long interspersed nuclear elements (LINEs) and short interspersed nuclear elements (SINEs) (adj. $r^{2}=0.53, p=0.0043$, Pearson's test corrected by the phylogeny), miniature inverted-repeat transposable elements (MITEs) (adj. $\mathrm{r}^{2}=0.40, p=0.021$, Pearson's test corrected by the phylogeny), and the genome size. This result suggested that the expansions of other TEs (LTRs, LINEs, SINEs, and MITEs) also contribute to genome size evolution in Hymenoptera.

In G. flavifemur, $40.3 \%$ of the genome consists of DNA transposons, whereas the DNA transposons contents in other hymenopteran insects are much lower $(0.2$ to $13 \%$ ) (Additional file 1: Supplementary Table 8). The DNA transposon expansion might be largely responsible for the genome size increase in G. flavifemur. To further investigate the main contributors of TE expansion, we identified four most abundant superfamilies of DNA transposons in the G. flavifemur genome: CACTA
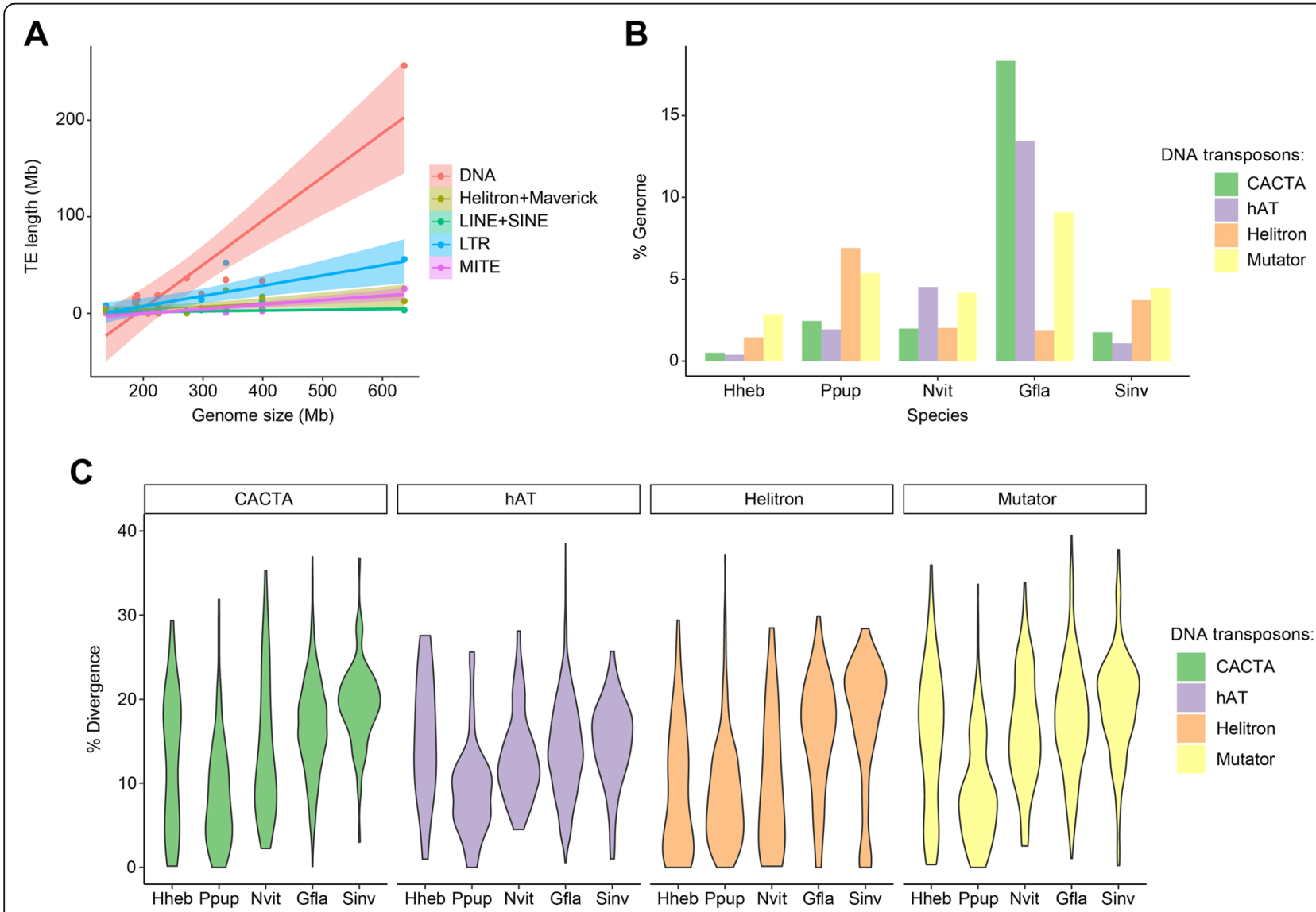

Fig. 3 DNA transposons in the genome of G. flavifemur. a Correlation between total coverage of different TE classes and genome size across Hymenoptera. Lines correspond to linear regressions; shadows correspond to the $95 \%$ confidence intervals around the mean predictions. $\mathbf{b}$ The content of top four superfamilies of DNA transposons among five Hymenoptera species. c Violin plots showing each DNA transposon's frequency distribution of sequence divergence level from the inferred ancestral consensus sequences. Gfla, G. flavifemur; Nvit, N. vitripennis; Ppup, P.

puparum. Hheb, H. hebetor; Sinv, S. invicta 
(18.3\%, Superfamily code: DTC), hAT (13.4\%, Superfamily code: DTA), Mutator (9.1\%, Superfamily code: DTM), and Helitron (1.9\%, Superfamily code: DHH) (Fig. 3b; Additional file 1: Supplementary Table 9). Although $P$. puparum has the highest Helitron content among five hymenopterans, the contents of CACTA, hAT, and Mutator superfamilies in the G. flavifemur genome were much higher than that in other genomes. We also found the divergence levels of these TE superfamilies are generally lower than $20 \%$ among five hymenopteran insects, suggesting that they may be recently active in the genomes (Fig. 3c). Compared with the other four genomes, G. flavifemur lacks shared patterns in terms of TE divergences. In addition, the closest relative of G. flavifemur that has been sequenced is Goniozus legneri (family Bethylidae, superfamily Chrysidoidea), with a small genome $(140.1 \mathrm{Mb})$ and low TE content (7.8\%; Additional file 1: Supplementary Table 8) [36]. However, the genome of G. legneri was poorly assembled with a scaffold N50 of $167.3 \mathrm{~kb}$, so this genome was not included in other analyses of the paper [36]. Thus, we concluded that the expansion of DNA transposons, mainly from CACTA, hAT, and Mutator superfamilies, occurred in G. flavifemur has predominantly enlarged its genome, after G. flavifemur diverged from G. legneri about 162 million years ago [1].

\section{Gene content comparison}

We identified 12,696 orthogroups (OGs) using OrthoFinder among G. flavifemur and the 12 other hymenopteran insects used in our analyses. Of these, 4608 OGs (5281 G. flavifemur genes) are present in all hymenopteran insects analyzed in this study. The G. flavifemur genome contains 727 Aculeate-specific genes, which is more than other aculeates. GO enrichment analysis revealed that these Aculeate-specific genes are enriched in DNA integration, DNA recombination, cellular aromatic compound metabolic process, and nitrogen compound metabolic process ( $p$ $<0.05$, false discovery rate adjusted, FDR-adjusted; Additional file 1: Supplementary Table 10). A total of 8453 genes from the G. flavifemur genome were species-specific and among which, 2516 showed BLAST hits against the NCBI nr database, suggesting that they may have homologs outside the phylogenetic context of our comparative genomics study. The rest of 5937 genes were inferred as species-specific and/or orphan in G. flavifemur. These 5937 genes were enriched in GO terms related to serine hydrolase activity and phosphorus-nitrogen bonds hydrolase activity $(p<0.05$, FDR-adjusted; Additional file 1: Supplementary Table 11).

Gene selection and gene family evolution in G. flavifemur Unlike most of the parasitoid wasps in the infraorder Parasitoida (e.g., braconid wasps and chalcid wasps), the dryinid wasps (only female wasps) are usually both parasitoids and predators on their hosts [8]. As parasitoids, females lay eggs on the live hosts, and their larvae hatch and feed on the host. As predators, females catch the hosts and feed on the hemolymph and tissues directly [8]. The hosts of dryinid wasps are from the suborder Auchenorrhyncha (order Hemiptera), most of which are planthoppers and leafhoppers. They are among the extremely agile insects, and adept at jumping and flying. To increase the success rate of parasitism and predation, female wasps are morphologically distinct from the males, including elongated and very mobile prothorax, a pair of chelae-like fore legs, and an ant-like, wingless body [4]. Predation behavior is universal in aculeate insects, such as ants, vespids, spider wasps, and dryinid wasps [5]. However, the predation mode of the dryinid wasps is different from most aculeates. Female dryinid wasp hunts and feeds directly on hosts (i.e., adult predacious feeding), while many aculeates paralyze their prey and transport it to their nests for larval feeding (i.e., provisioning predators) [5]. As a member of superfamily Chrysidoidea, dryinid wasp (family Dryinidae) represents the only case of predation, suggesting an independent origin of predatory behavior in Dryinidae. To gain insight into the genomic basis of the G. flavifemur-specific evolutionary traits, including the origin of predation, we performed genome-wide selection analysis and gene family evolutionary analysis to screen for gene changes that occurred in the G. flavifemur genome.

First, 2992 single-copy genes were used for screening the signatures of positive selection on the terminal branch of G. flavifemur in the phylogeny in Fig. 2a. Here, two tests (aBSREL model in HyPhy and branchsite model in PAML) were used ( $p<0.05$, FDR-adjusted) to define the significance of each candidate gene. Only those genes showing positive selection signals in both methods were used for the following study. In total, our strict screening criteria identified 183 genes that have evidence of positive selection in the G. flavifemur genome (Additional file 1: Supplementary Table 12). We did not observe any significantly enriched GO terms for these positively selected genes, suggesting that the functions of these genes might be diverse $(p>0.05$, FDRadjusted). We found some positively selected genes are related to mitochondrial functions, including NDUFB3, NDUFA9, mtTFB1, Myg1, and PTCD3 [37-40] (Additional file 1: Supplementary Table 12). Among them, two genes (NDUFB3 and NDUFA9) are in the mitochondrial electron transport chain (Complex I), which transfers electrons from NADH to ubiquinone [37]. Given the critical role of mitochondria in cellular respiration and energy production, these mitochondrial-related positively selected genes might function in providing energy for G. flavifemur's hunting. In addition, a number 
of positively selected genes are involved in actin cytoskeleton, muscle contraction, and motor activity, including TLN1 [41], TBCE [42], Scgß [43], SPG11 [44], ALS2 [45], TWF1 [46, 47], and SIM [48] (Additional file 1: Supplementary Table 12). These may also contribute to the predation behavior of G. flavifemur. Interestingly, our positively selected gene set includes a Piwi-like AGO3 gene, which belongs to the piRNA pathway. This AGO3 protein interacts with piRNA and plays a central role during meiosis by repressing TEs and preventing their mobilization, which is essential for germline integrity [49-52]. Our results above have indicated that TEs massively expanded in the G. flavifemur genome and some TEs may still be active. Thus, we hypothesize that this selected AGO3 of G. flavifemur might have functions on repressing TEs in the germline.

Additionally, we performed a rank-based branch length comparison method to study the protein evolutionary rates of the G. flavifemur branch. Amino acid substitutions provide strong evidence to fast or slow evolution when the divergence time is very long and the synonymous substitutions may be saturated. This method was used to identify rapidly evolving proteins in the Trichogramma wasp genome [14]. In this analysis, we reconstructed the phylogenetic tree of each of the 2992 single-copy proteins, and then for each protein, we extracted the total branch length and the terminal branch length of G. flavifemur. The total branch length represents the general protein evolution pattern of each protein, while the terminal branch length of G. flavifemur represents the protein evolution rate after G. flavifemur diverged from other species. Here, we first assigned ranks to proteins (from 1 to 2992) based on their total branch lengths (i.e., total branch length rank). Then, we assigned ranks to proteins (from 1 to 2992) based on their branch lengths of G. flavifemur branches (i.e., G. flavifemur branch length rank). We next binned proteins into groups of 300 based on their total branch length rank, which could avoid overrepresentation among any protein categories based on general evolutionary rates. In each bin, we selected the proteins with the top $10 \%$ largest discrepancy in rank between the G. flavifemur branch length rank and the total branch length rank, as rapidly evolving proteins (i.e., we chose the proteins with the top $10 \%$ largest values of the G. flavifemur branch length rank minus the total branch length rank) (Additional file 2: Supplementary Figure 4). We found that the rapidly evolving protein set is largely divergent from the positively selected gene set, with only 18 genes overlapped. In these rapidly evolving proteins, we found additional proteins involved in respiratory electron transport chain, which are ETFRF1 and NDUFB7 [37, 53]. Furthermore, additional proteins which may contribute to motor activities were found, including profilin [54],
cGMP-dependent protein kinase (cGK) [55], and twopore potassium channel protein sup-9 [56]. Four proteins from the $20 \mathrm{~S}$ core proteasome complex were identified as rapidly evolving proteins, suggesting a potentially important role of proteasomes in G. flavifemur. One of these four genes is also in the positively selected gene set. In addition, three $\mathrm{ABC}$ transporter proteins (related to detoxification), one visual system homeobox 2 protein (related to visual perception), and one cryptochrome-1 protein (related to circadian rhythm) were identified. Together, our searches for positively selected genes and rapidly evolving proteins revealed several genes or proteins involved in energy production and motor activity, which suggests that altered genes or proteins may contribute to the adaptation of predatory behavior in G. flavifemur.

We next investigated the gene family expansions and contractions in the G. flavifemur genome. A total of 434 gene families were expanded in G. flavifemur comparing to the common ancestor of the Aculeate, including chitinase-like proteins (glycosyl hydrolase family 18), neprilysins (zinc-dependent metalloproteases), trypsins, venom carboxylesterases, esterases, G-protein-coupled receptors, and several transcription factors (Fig. 2a). The significantly enriched GO terms included digestion and regulation of skeletal muscle adaptation, which may also associate with the predation behavior $(p<0.05$, FDRadjusted; Additional file 1: Supplementary Table 13). The GTF2IRD (general transcription factor II-I repeat domain-containing protein) genes were expanded in $G$. flavifemur, and they may contribute to slow-twitch fiber type specificity during myogenesis and regenerating muscles [57]. Interestingly, we found a number of expanded genes from chitinase-like gene family (glycosyl hydrolase family 18) and neprilysin family were much more highly expressed in the venom gland of G. flavifemur than in carcass (i.e., adult female tissues minus the venom gland), adult male, pupa, and larva, suggesting that they might be venom genes and play important roles in parasitoid-host interactions. See below for the detailed analyses about the chitinase/chitinase-like gene family and the neprilysin family.

\section{Venom gland-associated genes of G. flavifemur}

Among most of the hymenopteran insects, venom plays essential roles in their life. For example, parasitoid wasps use venom to manipulate the metabolism and immunity, and gene expression of the host to establish a suitable environment for wasp larvae [58-64], while some predatory hymenopterans (e.g., vespid wasps and ants) use venom for prey capture and defense $[63,65,66]$. The venom components of several parasitoid wasps and predatory hymenopterans have so far been reported [61, 65-70]. However, little is known about the venom components and their functions of G. flavifemur, which acts 
as both parasitoid and predator on its host. We hypothesize that the venom of G. flavifemur might have similar functions with the venoms of both parasitoid wasps and predatory wasps, and it is expected to play a key role in both altering host's metabolism and immunity (like many parasitoid wasps' venom), as well as paralyzing the host temporarily (like many predatory wasps' venom).

In order to gain insights into the putative venom gland-associated genes (VGGs) of G. flavifemur, we analyzed the RNA-seq datasets derived from venom gland and carcass (i.e., adult female tissues minus the venom gland) respectively. The venom gland is a highly specialized organ, which produces the venom components. We found that there is only a small set of genes highly expressed in the venom gland. For example, only 157 and 474 genes account for $80 \%$ and $90 \%$ of expression in the venom gland of G. flavifemur; however, 979 and 2297 genes account for $80 \%$ and $90 \%$ of expression in adult female (Additional file 2: Supplementary Figure $5 \mathrm{~A})$. This pattern could also be found in the venom gland of many other parasitoid wasps [61]. Based on the transcriptome data, we used three major criteria to identify VGGs in G. flavifemur, (1) VGGs must be among the top500 expressed genes (ranked by the median FPKM values) in the venom gland transcriptome; (2) VGGs must be significantly highly expressed in the venom gland relative to the carcass $(q<0.05)$; (3) VGGs must remain low expression levels in the carcass (median FPKM < 50). In total, 154 VGGs were identified in G. flavifemur (Additional file 1: Supplementary Table 14). These 154 VGGs have significantly higher expression levels in the venom gland and low expression levels in other developmental stages $(p<2.2 \mathrm{e}-16$, Wilcoxon rank-sum test; Additional file 2: Supplementary Figure 5B). Among these 154 VGGs, 32 genes have predicted signal peptides (Additional file 1: Supplementary Table 14), which indicates the presence of secretory signals and is considered as one of the characteristics of venom proteins in previous studies $[68,69]$. However, signal peptide may not be necessary for a venom protein, since evidence has shown that some RNA-seq supported proteins (encoded by venom gland highly expressed genes) without signal peptides can also be detected in venom proteomes [61, 68]. Moreover, venom-related extracellular vesicles (i.e., venosome) were found in some wasps, which may directly transport venom proteins to their targets in hosts, even if they do not contain signal peptides [71]. The VGGs contain a broad range of functional components, such as proteases and peptidases (46 genes, $29.9 \%$ ), protease inhibitor (1 gene, $0.7 \%$ ), lipases (10 genes, 6.5\%), chitinase-like genes (29 genes, 18.8\%), and oxidoreductases (3 genes, 1.9\%) (Additional file 2: Supplementary Figure 5C). Some of them are known venom proteins of other parasitoid wasps, including serine proteases, serpins (protease inhibitors), phospholipases, and major royal jelly proteins $[67,68]$.

Notably, a number of chitinase-like genes (29 genes, 8 genes with predicted signal peptides) were found to be specifically highly expressed in the venom gland of $G$. flavifemur (the mean FPKM in the venom gland is 4228.5, while the mean FPKM in the carcass is 5.4). Such a large number of venom-expressed chitinase-like genes was not reported in any other wasps, and might play an important role during the parasitism and hunting of G. flavifemur. It could occur due to the gene duplications after a single venom gene recruitment, or many independent venom gene recruitment events after gene duplications, or a complex evolutionary history that includes both cases above. Additionally, the venom gene recruitment of chitinase-like genes might be due to the lateral gene transfer (LGT), and it has been reported in some parasitoids in Chalcidoidea [72]. However, we did not find any evidence to support the hypothesis that some of these highly expressed chitinase-like genes in the venom gland were laterally transferred from bacteria or fungi.

Because of the abundant chitinase-like genes and clotting-related proteases (8 genes) in the venom gland, the GO enrichment analysis showed that the VGGs are enriched in carbohydrate metabolic process, imaginal disc development, coagulation, wound healing, proteolysis, cuticle development $(p<0.05$, FDR-adjusted; Additional file 1: Supplementary Table 15 and Additional file 2: Supplementary Figure 5D). It seems that the venom of G. flavifemur might regulate the host's cuticle development and prevent the host from dying due to excessive hemolymph loss. Parasitized hosts will be alive until the mature wasp larvae leave the hosts for cocooning and pupation [4]. Therefore, it is reasonable to hypothesize that G. flavifemur venom might be involved in the wound healing process to ensure the host is alive.

There are total 26 neprilysin-like genes (M13 peptidases) in the VGGs, and 7 of them contain predicted signal peptides (Additional file 1: Supplementary Table 14). Neprilysin is a zinc-dependent metalloprotease with a broad range of physiological targets, including natriuretic, vasodilatory, and neuro peptides [73]. Venom neprilysins have been reported in many venomous animals, such as jellyfishes, snakes, spiders, and solitary hunting wasps Eumenes decorates and Ampulex compressa [70, 74-78]. The functions of venom neprilysins are related to neurotoxicity which can paralyze the prey immediately $[79,80]$. Such high abundant venom neprilysin-like genes may imply that paralysis might be one of the major functions of the venom of G. flavifemur. Moreover, we found a hemolymph lipopolysaccharidebinding protein gene in the VGG set, which may play a 
role in bacterial clearance and protect parasitoid larvae from the influence of bacteria [81]. The hemipteran hosts of G. flavifemur usually carry some intracellular symbionts [82]. It would be interesting to study if this venom lipopolysaccharide-binding protein could target the host's bacterial symbionts.

\section{Expansion and venom expression of chitinase-like genes in G. flavifemur}

To gain more insights into the expansion of the chitinaselike gene family (glycoside hydrolase 18 family, GH18) in G. flavifemur, we searched GH18 domain-containing proteins in 17 insect genomes, which includes 13 hymenopteran genomes (including G. flavifemur), 1 lepidoptera genome (Bombyx mori), 1 coleopteran genome (Tribolium castaneum), 1 diptera genome (Drosophila melanogaster), and 1 hemipteran genome (Acyrthosiphon pisum). The four non-hymenopteran genomes we analyzed here allow us to determine the ancestral state of chitinase-like gene family of Hymenoptera. In total, 260 GH18 domaincontaining proteins were found and 61 of them were in the G. flavifemur genome, representing the most abundant one when compared to the other 16 genomes. Maximum likelihood phylogenetic analysis indicated that the chitinase-like genes in group 4 (7 genes) and group 5 (47 genes) were expanded in the G. flavifemur genome, whereas the gene counts in other chitinase groups among 17 insect genomes were highly conserved (Fig. 4a, b).

Group 5 chitinase-like proteins are also annotated as imaginal disc growth factors (IDGFs), which are a small family of chitinase-related secretory proteins found in many insects [83-87]. They lack chitinase activity due to an amino acid substitution of a key glutamate residue (E) in a conserved active site motif [88]. However, a few examples in T. castaneum and parasitoid wasp Microplitis mediator and Cotesia chilonis show that although IDGFs retain the glutamate residue in the active site motif, a D to A substitution in the same motif (from DxxDxDxE to DxxDxAxE) also results in the loss of function of IDGFs (Additional file 2: Supplementary Figure 6) [88]. In G. flavifemur, we identified 47 Idgf genes. This number is much higher than any other insects we surveyed, including G. legneri, the closest relative of $G$. flavifemur (Additional file 1: Supplementary Table 16). Phylogenetic analysis showed that Idgf genes were largely expanded in the G. flavifemur genome, and 44 of total 47 Idgf genes were clustered together (Fig. 4b). We found a well-supported (87\% of rapid bootstraps) insect conserved Idgf clade, which contains three G. flavifemur Idgf genes. In total, 44 Idgf paralogs in the G. flavifemur genome clustered together to form a G. flavifemur-specific clade instead of clustered with the Idgf ortholog in insect conserved Idgf clade, suggesting a rapidly evolutionary history of these paralogs (Fig. 4b). Gene distribution analysis revealed many Idgf gene tandem arrays (with 2 or more Idgf genes) in the G. flavifemur genome, indicating the tandem duplications of Idgf genes. The largest tandem array contains 6 Idgf genes (Fig. 4c). Gene expression patterns showed that the three G. flavifemur Idgf genes in insect conserved Idgf clade expressed widely across development, while many Idgf genes in G. flavifemur-specific clade display strong venom-gland biased expression patterns and lowly express in adult male, pupa, and larva (Fig. 4c). Due to the low pairwise identity, with only $27.22 \%$ on average, we concluded that the cross-mapping events among these genes are neglectable during RNA-seq analysis. Interestingly, we observed an Idgf tandem array, which includes four genes. Three of them are insect conserved Idgfs (no venom expression) while the last one is G. flavifemurspecific. Moreover, this G. flavifemur-specific Idgf displayed a strong venom-gland biased expression pattern. Among 47 Idgf genes, 28 were assigned as VGGs in our analysis (Fig. 4b, c). Gene family expansion and venom recruitment of Idgf genes in G. flavifemur implied that these genes may have important functions as venom components, although only 12 of 28 venom glandassociated Idgf genes have predicted signal peptides. Venom IDGF proteins are unusual in hymenopterans and have been reported in only two additional parasitoid wasps, M. mediator and Chelonus inanitus [89, 90]. These venom IDGF proteins (MmV234 and Ci-48b) were clustered with other hymenopteran IDGFs in the insect conserved Idgf clade (Fig. 4b).

An additional gene from the group 4 chitinase subfamily was also highly expressed in the venom gland and very lowly expressed across development (Fig. 4c). This gene is located in a tandem array of group 4 chitinase genes, showing a tandem duplication event. Together, our observations imply that the expanded Idgf and group 4 chitinase genes with venom-gland high expressions might have important functions in G. flavifemur-host interaction. Further studies are needed to investigate the function of these venom chitinase-like genes.

\section{Expansion and convergent venom recruitment of neprilysin-like genes in G. flavifemur}

In the G. flavifemur genome, we identified 66 neprilysinlike genes (containing Peptidase_M13 domain), which is much more abundant than any other insects in this study, ranging from 5 to 42 (Additional file 1: Supplementary Table 17). Phylogenetic analysis further confirmed that neprilysin-like genes were largely expanded in the G. flavifemur genome, and 41 neprilysin-like genes were clustered together to form a G. flavifemurspecific clade (Fig. 5a, b). Notably, this G. flavifemur-specific clade includes 23 of total 26 venom glandassociated neprilysin-like genes defined by the venom 


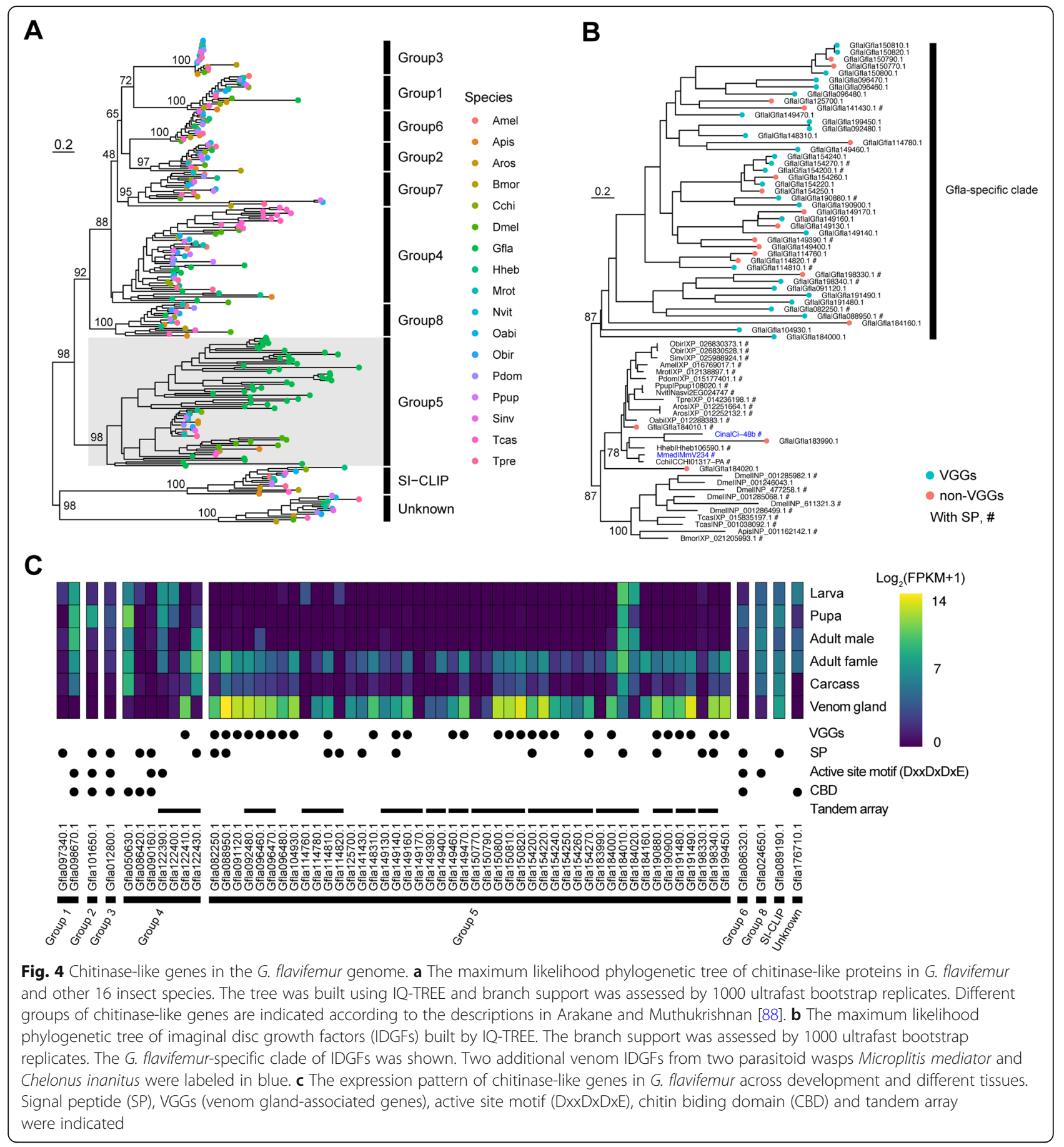

gland transcriptomes (Fig. 5b). Neprilysin is a zincdependent metalloprotease and is predicted to metabolize regulatory peptides in prey's nervous system and paralyze the prey as a venom protein $[79,80]$. The expansion and venom high expression of these neprilysin-like genes suggest that the G. flavifemur venom might have a powerful paralyzing effect on the host. Additionally, we identified a large expansion of the Orussus abietinus neprilysin-like genes (Fig. 5a). O. abietinus is an ectoparasitoid of xylobiontic larvae of beetles or wood wasps [35]. In phylogeny, O. abietinus represents the closest relative of Apocrita, which may have a similar lifestyle with the parasitoid ancestor [2]. It is worth investigating whether the expanded neprilysinlike genes are also highly expressed in the venom of $O$. abietinus.

Venom neprilysins have been reported in many venomous animals, such as jellyfishes, snakes, and 


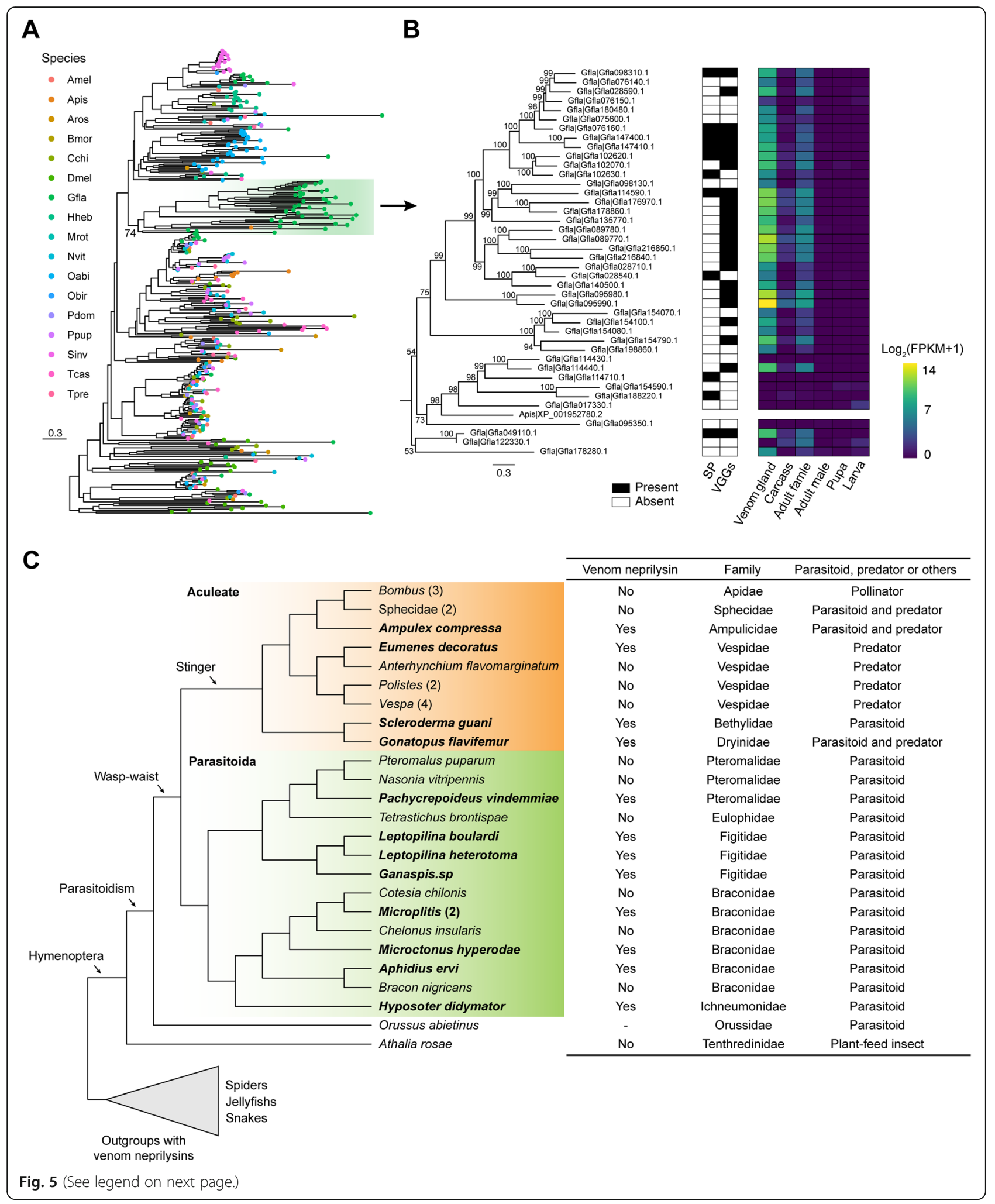


(See figure on previous page.)

Fig. 5 Neprilysin-like genes in the G. flavifemur genome. a The maximum likelihood phylogenetic tree of neprilysin-like proteins in G. flavifemur and other 16 insect species. The tree was built using IQ-TREE and branch support was assessed by 1000 ultrafast bootstrap replicates. b The phylogenetic tree of neprilysin-like genes in the G. flavifemur-specific clade and their expression pattern in venom gland, adult female tissues minus the venom gland (i.e., carcass), adult female, adult male, pupa, and larva. SP, signal peptide; VGGs, venom gland-associated genes. c Venom recruitment of neprilysin-like genes in Hymenoptera. Totally, the venom components of 31 hymenopterans were exanimated (15 in Parasitoida and 16 in Aculeate). The ectoparasitoid wood wasp O. abietinus and the non-venom basal hymenopteran A. rosae were also included. The phylogeny of these hymenopterans was obtained from previous studies $[2,91]$. Others animals, such as snakes, spiders, which carry venom neprilysin-like genes were shown

centipedes and spiders, suggesting many convergent venom recruitments $[74,76,78,80]$. We next asked whether the neprilysin-like genes are present in the venoms of other species in Hymenoptera by convergent recruitments. To this end, we examined the reported venom components of 30 additional hymenopterans (15 in Parasitoida and 15 in Aculeate) to search venom neprilysin-like genes (Fig. 5c). In total, including G. flavifemur, we found venom neprilysin-like genes in 13 wasps (9 in Parasitoida and 4 in Aculeate). In Parasitoida, venom neprilysin-like genes were found in a chalcid wasp Pachycrepoideus vindemmiae, and 3 figitid wasps Leptopilina boulardi, L. heterotoma, and Ganaspis sp., 4 braconid wasps M. mediator, M. demolitor, Microctonus hyperodae, Aphidius ervi, and an ichneumonid wasp Hyposoter didymator. In Aculeate, we found additional venom neprilysin-like genes in a bethylid wasp Scleroderma guani, a solitary hunting wasp Eumenes decorates and an emerald cockroach wasp Ampulex compressa. We then summarized the presence or absence of the venom neprilysin-like genes in total 31 hymenopterans and mapped the information to their phylogeny. Our results show that the recruitments of venom neprilysin-like genes are mainly scattered throughout the Hymenoptera phylogeny. This indicates that most recruitment events of venom neprilysin-like gene occurred independently during the radiation of Hymenoptera (i.e., convergent venom recruitments).

\section{Gene expression changes in female adults after feeding on the preys}

Some female insects, such as blood-feeding mosquitoes, need to feed on proteins to trigger egg development [92]. A previous study reported that female dryinid wasps often consumed the first host captured in the day [5]. We then hypothesized that the hunting and prey feeding behaviors of $G$. flavifemur female adults may be beneficial to reproduction. To test our hypothesis, we analyzed the transcriptome data derived from the preyfeeding females (protein-rich diet) and sucrose-feeding females to characterize differential expressed genes between these two treatments. In total, 24 genes were significantly upregulated in the prey-feeding females when compared to the sucrose-feeding females (fold change >
4 and $q<0.05$; Additional file 1: Supplementary Table 18). We observed that 3 yellow genes were significantly activated (5.44-8.09-fold higher expression) after feeding on the preys (Fig. 6). Compared with other insect yellow genes, phylogenetic analysis suggested that these 3 activated yellow genes belong to subfamily yellow-g, yellow$g 2$, and yellow- $h$, respectively (Additional file 1: Supplementary Table 19). Yellow genes are common within arthropods and are homologous with major royal jelly protein encoding genes $[93,94]$. In some well-studied insects, such as fruit fly, mosquito, and silkworm, some members of the yellow gene family are associated with reproductive maturation [95-97]. In G. flavifemur, analyses of transcriptome data of male and female adults showed that the 3 upregulated yellow genes in the preyfeeding females are extremely female-biased genes, with 266.98-1656.33-fold higher expression in the female adults than in the male adults $(q<0.05$; Additional file 1: Supplementary Table 19). Therefore, our finding indicates that 3 female-biased yellow genes are significantly upregulated in females after feeding on the preys, and these genes may be involved in the reproduction of $G$. flavifemur. Other significantly upregulated genes in the prey-feeding females including trypsin-1, lipase 3, neutral ceramidase, takeout, and fatty acyl-CoA reductase (Fig. 6 and Additional file 1: Supplementary Table 18). We also identified 20 significantly downregulated genes in the prey-feeding females when compared to the sucrose-feeding females, which included maltase2, growth arrest-specific protein 8, liver carboxylesterase, and small heat shock protein $\mathrm{C} 4$ (fold change $>4$ and $q$ $<0.05$; Additional file 1: Supplementary Table 20).

\section{Sex-biased genes and male-biased opsin genes in G. flavifemur}

Due to the obvious sexual dimorphism of G. flavifemur, we also investigated the sex-biased genes in this wasp by comparing the RNA-seq data of adult males and females. The 461 extremely female-biased genes (fold change > 16 and $q<0.05)$ were enriched in nucleosome assembly, meiotic cell cycle process $(p<0.05$, FDR-adjusted; Additional file 1: Supplementary Table 21). However, the 362 extremely male-biased genes (fold change $>16$ and $q<0.05)$ showed a striking enrichment of GO terms 


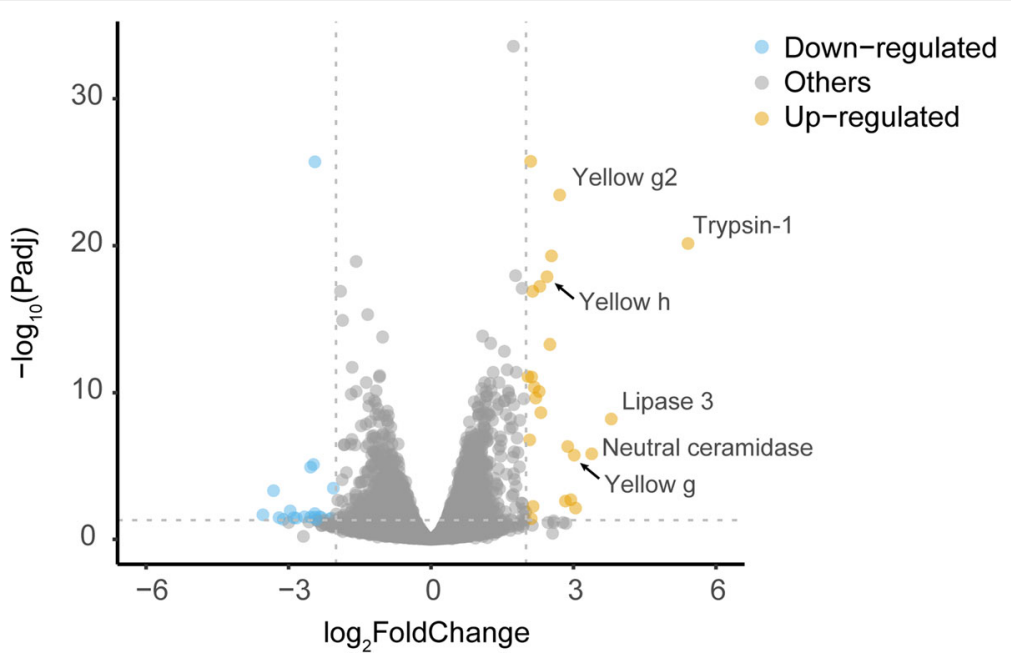

Fig. 6 Volcano plot showing differential gene expression between prey-feeding females and sucrose-feeding females of G. flavifemur. In total, 24 significantly upregulated genes (shown in yellow) and 20 significantly downregulated genes (shown in blue) were found (fold change $>4$ and $q$ $<0.05$ ). Importantly, three yellow genes (yellow-g, yellow-g2, and yellow-h) significantly increased their expressions (5.44-8.09-fold higher expression) after feeding on the preys

associated with sensory perception, detection of stimulus, and cell-cell signaling ( $p<0.05$, FDR-adjusted; Additional file 1: Supplementary Table 22). The significant activation of the sensory perception system of the adult males might be due to their short life (only about 2 days), while females usually live longer (about 20 days). Within the short lifespan of males, they need to find females to mate in a limited time frame; thus, they are reasonable to have a powerful sensory perception system. We identified 7 olfactory receptor genes with higher expression (21.14-415.75-fold higher) in the males than in the females. These genes might be related to locating the females for mating. The adult male has wings; however, the adult female is a wingless, ant-like wasp. Thus, as expected, a flightin gene that is involved in regulating flight muscle contraction, was 161.87 -fold highly upregulated in the adult male than in the females (Additional file 1: Supplementary Table 23).

We next analyzed the opsin genes, which are important in insect visual perception [98]. Our comparison among 12 hymenopteran insects showed that the ultraviolet (UV)-sensitive opsin and the blue-sensitive opsin are both kept as a single copy in all 12 hymenopterans. The paper wasp Polistes dominula has three long-wave sensitive (LWS) opsin genes, while the rest species have two LWS-opsin copies (Fig. 7a). Comparative transcriptome analysis among G. flavifemur, $P$. puparum, and $N$. vitripennis revealed that Blue-opsin, $U V$-opsin, and LWS1-opsin in the three wasps are male-biased genes, with 1.89-4.02-fold higher expression in the males than in the females $(q<0.05$; Fig. 7b). Surprisingly, LWS2opsin of G. flavifemur was 86.86-fold higher upregulated in the males compared to females $(q=9.72 \mathrm{e}-106)$, while the LWS2-opsin of $P$. puparum was only 2.89 higher expressed in the males $(q=2.24 \mathrm{e}-06)$. In addition, the LWS2-opsin of $N$. vitripennis did not show a male-biased expression pattern $(q=0.305$; Fig. 7b). Taken together, our transcriptomic comparisons among G. flavifemur, $P$. puparum, and $N$. vitripennis indicate a global malebiased expression pattern of Blue-opsin, UV-opsin, and LWS-opsin, except the LWS2-opsin of N. vitripennis, and an extremely male-biased LWS2-opsin gene in G. flavifemur. The male-biased expression of these opsin genes may contribute to partner searching and mating.

\section{Discussion}

Herein, by combining both Nanopore long-read sequencing and Illumina short-read sequencing strategies, we generated a high-quality reference genome of the pincer wasp G. flavifemur, which is the first genome sequence of the family Dryinidae. This species resides a key evolutionary position in Hymenoptera, as the early branch of the sting wasps (Aculeate), providing a valuable resource to facilitate our understanding of Hymenoptera evolution. In addition, G. flavifemur has many unique biological characteristics, including parasitism, predation, and sexual dimorphism.

In this study, our comparative genomics analysis highlighted a number of positively selected genes and rapidly evolving proteins involved in energy production and motor activity, which may play roles in the predatory adaptation of G. flavifemur. These findings expand our understanding of the hunting behavior evolution in Hymenoptera. By incorporating transcriptomic data, we found that 3 yellow genes (yellow-g, yellow-g2 and yellow-h) were significantly activated in 

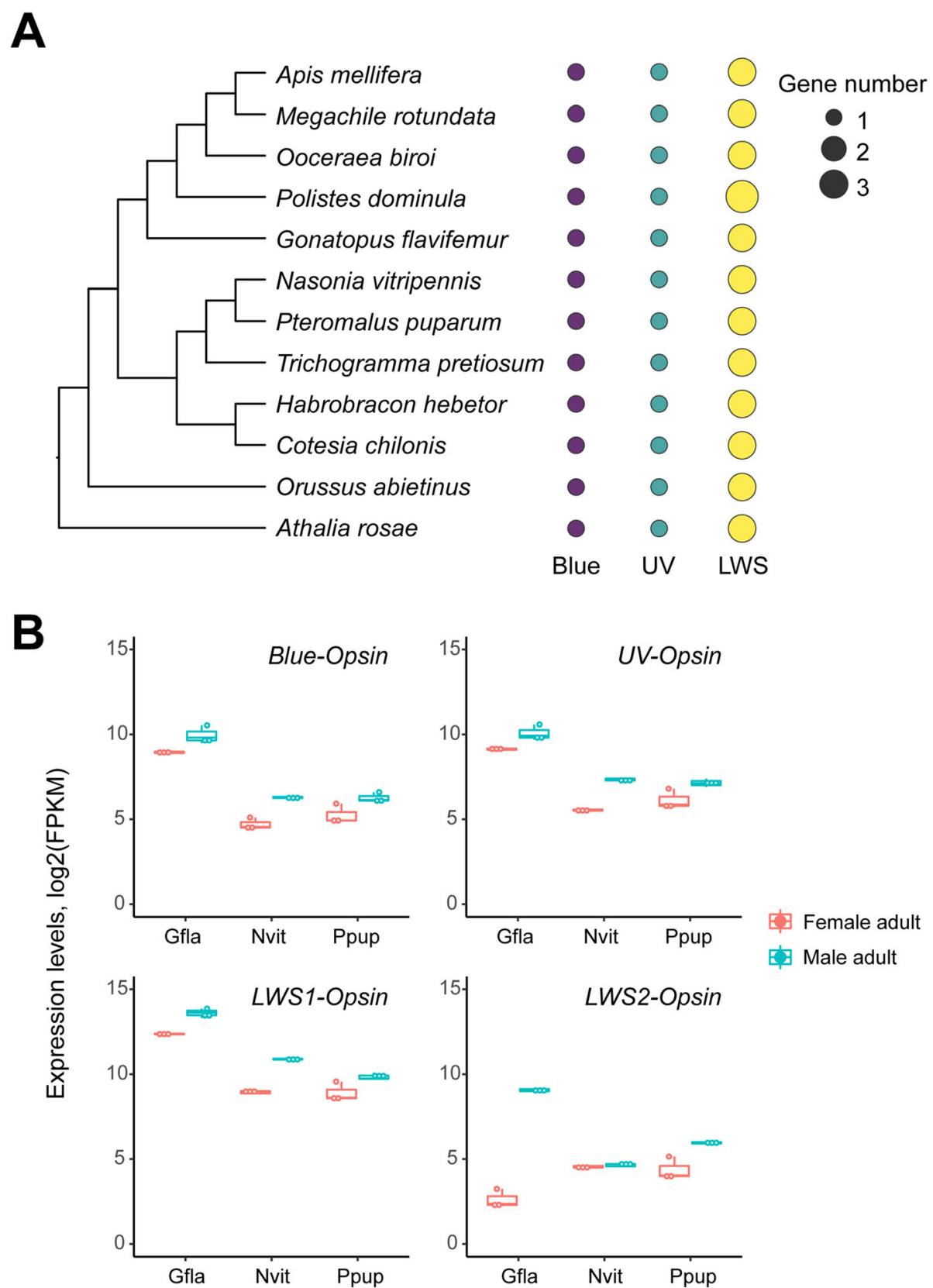

Fig. 7 Opsin genes in G. flavifemur. a Opsin genes in the 12 hymenopteran insects. Bubble plot indicating the gene count of each opsin gene. Blue, short wavelength-sensitive opsin; UV, ultraviolet-sensitive opsin; LWS, long-wavelength-sensitive opsin. $\mathbf{b}$ Differential gene expression of opsin genes between female adult and male adult in three parasitoid wasps. The Blue-opsin, UV-opsin and LWS1-opsin in the three wasps are male-biased genes, with 1.89-4.02-fold higher expression in the males than in the females $(q<0.05)$. The LWS2-opsin of G. flavifemur was 86.86fold higher male-biased expressed $(q=9.72 \mathrm{e}-106)$. The LWS2-opsin of $P$. puparum was 2.89 higher expressed in the males $(q=2.24 \mathrm{e}-06)$. However, the LWS2-opsin of N. vitripennis did not show any sex-biased expression pattern $(q=0.305)$. Gfla, G. flavifemur; Nvit, $N$. vitripennis; Ppup, P. puparum

female G. flavifemur after feeding on the preys. It has been reported that some members of the yellow gene family are associated with reproductive maturation. For example, yellow-g and yellow-g2 play a femalespecific role in the egg development of Drosophila and Aedes $[95,96]$. Moreover, all the yellow genes of silkworm Bombyx mori have a high transcription level in both ovary and testis, suggesting their reproduction-related functions [97]. Therefore, our discovery bridges the gap between the unique hunting behavior and reproductive advantages in the parasitoid wasp $G$. flavifemur. 
Venoms are key evolutionary innovations in most hymenopteran insects and have been used for predatory, defensive, and reproductive purposes. Our comprehensive study on the venom gland identified significant expansions and unique expression patterns of Idgf genes and neprilysin-like genes. The functions of Idgf genes in insects are diverse, and they have been proved to participate in cuticle formation, wing development, larval and adult molting, immune response, antimicrobial response, and hemolymph clotting $[88,99,100]$. In addition, IDGF proteins are present in mosquito saliva and may contribute to the modulation of the mammalian host response and enhancing mosquito-borne Zika virus infection [101]. Here, we hypothesize that these IDGF proteins in the venom of G. flavifemur might play various potential roles, for example, regulating the development of the hosts, participating in the wound healing of the hosts, and immune-related functions against the hosts and bacteria. On the other hand, neprilysin specializes in the role of metabolizing and regulating molecules in the mammalian nervous systems; for example, it can inactivate peptide transmitters and their modulators $[79,102]$. The expansion and venom high expression of neprilysinlike genes imply the G. flavifemur venom might have a powerful paralyzing effect on the host, which is of significant benefit to hunting and parasitism. Indeed, the temporary paralyzing effect of dryinid wasp's venom has been reported before [3, 103], and our discovery of neprilysin expansion could explain the molecular basis of venom function. By comparing 31 hymenopterans, our study further indicated that venom neprilysins are prevalent in many hymenopterans and the recruitment events of venom neprilysin-like gene occurred independently during the radiation of Hymenoptera (i.e., convergent venom recruitments). However, this is only the tip of the iceberg, and exploring the bigger picture of venom neprilysin evolution in Hymenoptera requires further indepth studies and whole-genome sequencing.

The genome size of G. flavifemur is much larger than most hymenopterans. The expansion of TEs, especially from DNA transposons, is a major contributor to the genome size enlargement in G. flavifemur. TE expansions and insertions can cause a variety of changes in the host genome, such as chromosomal rearrangements, gene disruptions, and gene expression regulations, some of which may be of benefit to adaptation [30, 104-107]. Several studies have shown that TE insertions play an essential role in helping insects to increase their adaptability, including raising the insecticide resistance and enhancing the ability to adapt to climate changes [108, 109]. In addition, $T E$ is an important factor in insect antiviral immunity and aging regulation [110]. We therefore hypothesize that the expansion of TEs and the accompanied TE insertions might contribute to the adaptation of G. flavifemur. We envision further investigations on how TE expansion benefits the adaptation of G. flavifemur.

\section{Conclusions}

Parasitoid wasps in the family Dryinidae display several interesting characteristics, such as the predatory behavior of adult females and the distinct sexual dimorphism. In this study, we present the genome of the dryinid wasp G. flavifemur to understand the genetic basis of these key innovations. Compared to other hymenopterans, our findings highlight that the TEs, especially DNA transposons, have massively expanded in the G. flavifemur genome, resulting in the genome size enlargement. Our genome-wide screens locate a number of positively selected genes and rapidly evolving proteins involved in energy production and motor activity, which may contribute to the predatory adaptation of G. flavifemur. We also show that 3 female-biased, reproductive-associated yellow genes in adult females expressed significantly higher after feeding on the preys, which may be beneficial to the egg production. This may explain the advantage of their unique predatory behavior. In addition, our transcriptomic analyses and following gene family analyses reveal the unique venom characters of G. flavifemur, such as the expansions of venom Idgf-like genes and neprilysin-like genes. Furthermore, we identify sexbiased genes based on the differences of gene expression between adult females and males, and observe an extremely male-biased LWS2-opsin gene in G. flavifemur. These results advance our understanding of the genome architecture, predatory adaptation, venom gene evolution, and sex-biased genes in G. flavifemur, and stimulate further comparative analyses of hymenopterans.

\section{Methods \\ Insects}

The dryinid wasps G. flavifemur were provided by Dr. Qiang $\mathrm{Fu}$ from his laboratory. The wasp colony was originated from about 50 wasps of a field population, which was collected at Hangzhou, China, in 2018. The colony of the hosts $N$. lugens was first collected from rice fields at Hangzhou, China, in 2018. Both parasitoid wasps and the hosts were maintained in the laboratory. The $N$. lugens was reared continuously on a susceptible rice variety (Taichung Native $1, \mathrm{TN} 1$ ) under laboratory conditions at $28 \pm 1{ }^{\circ} \mathrm{C}, 65 \pm 5 \%$ relative humidity (RH), $3500 \sim 4000$ Lux and a photoperiod of 16: $8 \mathrm{~h}$ (light: dark) [111]. The parasitoid wasps G. flavifemur were reared on 4-5th instar brown planthoppers under the same conditions. The details of samples for genome and transcriptome sequencing are summarized in Additional file 1: Supplementary Table 24. 


\section{Genome sequencing}

We applied the Nanopore and Illumina HiSeq X Ten platforms to sequence the genome of G. flavifemur. High-quality genomic DNA for de novo sequencing was extracted from 50 haploid male pupae using the sodium dodecyl sulfate (SDS)-based DNA extraction method followed by purification with VAHTS DNA Clean Beads (Vazyme, Cat. \# N411-03) according to the standard procedure provided by the manufacturer. The quality and concentration were then assessed by $1 \%$ agarose gel electrophoresis, NanoDrop ${ }^{\text {Ts }}$ One UV-Vis spectrophotometer (Thermo Fisher Scientific, USA) and Qubit ${ }^{\bullet} 3.0$ Fluorometer (Invitrogen, USA). For long library preparation, qualified DNA was size-selected (>10 kb) using the BluePippin system (Sage Science, USA), then two long libraries were processed according to the Ligation Sequencing Kit (SQK-LSK109, Oxford Nanopore) protocol and sequenced on two flow-cells using the PromethION sequencer (Oxford Nanopore). A short pairedend library with an insert size of 350 bp was constructed using a TruSeq Nano DNA HT Sample Preparation Kit (Illumina), and then sequenced on the HiSeq $\mathrm{X}$ Ten platform.

\section{Evaluation of genome size}

The paired-end Illumina reads were firstly filtered by fastp v0.20.0 [112]. Clean reads were used for estimating the genome size and heterozygosity using GenomeScope v1.0.0 [113] based on the 17-mer distribution analyzed by Jellyfish v2.3.0-1 [114]. The estimated genome size was further validated by flow cytometry following the standard procedure reported in $\mathrm{He}$ et al. [115]. Briefly, the heads of 20 adult insects were completely homogenized in $500 \mu \mathrm{L}$ icecold Galbraith's Buffer (45 mM $\mathrm{MgCl}_{2}, 20 \mathrm{mM} 3-\mathrm{N}$ morpholinopropane sulfonic acid, $30 \mathrm{mM}$ sodium citrate, and $0.1 \%$ (vol/vol) Triton X-100; pH 7.0). The homogenate was then filtered into a $1.5-\mathrm{mL}$ Eppendorf tube using 38- $\mu \mathrm{m}$ nylon mesh. To remove RNAs, RNase A (Takara, Japan) was added to the homogenate (final concentration of $20 \mu \mathrm{g} / \mathrm{mL}$ ) and incubated at $25^{\circ} \mathrm{C}$ for $10 \mathrm{~min}$. The precipitates were collected by centrifuging at $1000 \mathrm{~g}$ for $5 \mathrm{~min}$, and then suspended with $400 \mu \mathrm{L}$ phosphate buffer (pH 7.4) and stained with $50 \mu \mathrm{g} / \mathrm{mL}$ propidium iodide stock solution in darkness at $4{ }^{\circ} \mathrm{C}$ for $10 \mathrm{~min}$. Each sample was analyzed using the $\mathrm{MoFlo}^{\mathrm{m}}$ XDP High Speed Cell Sorter and Analyzer (Beckman Coulter, CA, USA) under 488-nm wavelength. Summit Software (Beckman Coulter, CA, USA) was used to obtain the nuclei peaks. The genome size was then estimated based on the outputs, using D. melanogaster as a control (Additional file 2: Supplementary Figure 2).

\section{Genome assembly and assessment}

We firstly used NextDenovo v1.1.1 (https://github.com/ Nextomics/NextDenovo) to correct Nanopore reads with -seed_cutoff $=10 \mathrm{k}$ parameter. The corrected reads were then assembled into primary contigs using wtdbg2 v2.3 [116]. Then, NextPolish v1.0.5 [117] was used to polish the assembly with both Nanopore long reads (three iterations) and Illumina reads (four iterations). Finally, we removed the bacterial contaminating contigs using the pipeline of Wheeler et al. [11]. Briefly, each contig was firstly split into $1000 \mathrm{bp}$ units, which were then searched against a bacterial genome database provided in Olafson et al. [15] using BLASTN v2.8.1 [16] (-evalue 1e-5). Contigs were identified as likely bacterial contigs if the proportion of bacterial matched units along their total number of units was larger than $40 \%$. BUSCO v5 [118] was used to assess the genome assembly completeness with the insect protein set (insecta_odb10). We also mapped the Illumina genomic reads and RNA-seq reads to the genome assembly by BWA v0.7.17 [119] and HISAT2 v2.2.1 [120] respectively. The mapping rates were counted by SAMtools v1.10 [121].

\section{Transcriptome sequencing and analysis}

RNA-seq libraries (insert size of $250 \mathrm{bp}$ ) were prepared from larva (7-day-old larvae, 10 individuals), pupa (10 individuals), female adult (1-day-old, 10 individuals), male adult (1-day-old, 10 individuals), venom gland (5day-old female adult, 100 individuals), and carcass (5day-old female adult, remove venom gland, 10 individuals). In addition, to investigate the gene expression changes between prey-feeding females and none preyfeeding females, six RNA-seq libraries (insert size of 250 bp) were prepared from the female adults (5-day-old) reared on $20 \%$ sucrose water and brown planthoppers (the preys), respectively. Three biological replicates were prepared for each sample. The transcriptomes were sequenced using the Illumina HiSeq X Ten platform with paired-end libraries. Raw reads from the RNA-seq were filtered using Trimmomatic v0.38 [122]. The clean reads were mapped to the genome assembly using HISAT2 v2.2.1 [120] and then assembled into transcripts using StringTie v2.1.0 [123]. RSEM v1.3.3 [124] was used for estimating the gene expression level, and DESeq2 package v1.30.1 [125] was used to perform the differential expression analyses.

\section{Repeat annotation}

We used the Extensive de novo TE Annotator (EDTA) pipeline to construct TE libraries for each species [126]. Briefly, we firstly applied a series of structure-based TE classification tools in the EDTA pipeline to identify each type of TE (LTR_FINDER [127], LTRharvest [128] and LTR_retriever [129] for LTR retrotransposons, TIR- 
Learner [130] and HelitronScanner [131] for DNA transposons). Outputs from each program were filtered by the EDTA pipeline (the filtering step was detailly described in Ou et al. [126]). Next, RepeatModeler v2.0 [132] was used to identify non-LTR retrotransposons and any unclassified TEs that were missed by the TE annotators above. Finally, all results were compiled into a comprehensive non-redundant TE library for downstream analysis. RepeatMasker v4.0.7 [133] was then used to search for known and novel TEs by mapping sequences against the de novo TE library and Repbase library v16.02 [134]. Tandem repeats were annotated using Tandem Repeat Finder v4.09 [135]. The divergences of each TE family were reported by RepeatMasker and then converted to nucleotide distance measures using the Jukes-Cantor nucleotide model to correct for multiple hits. Final results were pooled into bins of single unit distances, which recapitulates the history of TE class proliferation.

\section{Gene annotation}

Three approaches, as incorporated in the EVidenceModeler pipeline (EVM, v1.1.1) [136], were used to predict the protein-coding genes: de novo gene prediction, homology-based and RNA-seq-based approaches. For de novo gene prediction, we utilized AUGUSTUS v3.1 [137] and SNAP v2006-07-28 [138] to analyze the repeat-masked genome. For homology-based predictions, the protein sequences of invertebrates were retrieved from NCBI Reference Sequence Database as templates. Exonerate v2.2.0 (https://www.ebi.ac.uk/ about/vertebrate-genomics/software/exonerate) and GenomeThreader v1.7.1 [139] were used to align the reference proteins to the genome assembly and predict gene structures. For RNA-seq-based gene prediction, we used the transcriptome assembled from RNA-seq alignments and identified the candidate coding region of each transcript by TransDecoder v5.5.0 (https://github.com/ TransDecoder/TransDecoder). Finally, EVidenceModeler v1.1.1 [136] was used to integrate the genes predicted by the above three approaches and generate a consensus gene set. Gene Ontology (GO) analysis was carried out using the software Blast2GO v5.2 [140]. We next mapped the gene set to Kyoto Encyclopedia of Genes and Genomes (KEGG) pathways using BlastKOALA v2.2 [141] online service. We also searched the protein sequences in the SwissProt and TrEMBL databases using BLASTP v2.8.1 [16] (-evalue 1e-5).

\section{Comparative genomics}

We comprehensively considered three factors of each species for comparative genomics analyses and phylogenomic analyses, including genome quality, popularity, and evolutionary position. OrthoFinder v2.5.1 [20] was used to identify the orthologous and paralogous genes of 13 Hymenoptera genomes including O. abietinus (RefSeq assembly accession: GCF_000612105.2), C. chilonis (http://www.insect-genome.com/), Habrobracon hebetor (Ye et al. [142]), Trichogramma pertiosum (RefSeq assembly accession: GCF_000599845.2), Nasonia vitripennis (OGS2.0, Rago et al. [143]), P. puparum (OGS1.0, Ye et al. [13]), G. flavifemur (this study), P. dominula (RefSeq assembly accession: GCF_001465965.1), Solenopsis invicta (RefSeq assembly accession: GCF_000188075.2), Ooceraea biroi (RefSeq assembly accession: GCF_003672135.1), Megachile rotundata (RefSeq assembly accession: GCF 000220905.1), A. mellifera (RefSeq assembly accession: GCF_003254395.2), and A. rosae (RefSeq assembly accession: GCF_000344095.2). The basal hymenopteran $A$. rosae was used as an outgroup.

\section{Phylogenetic analysis}

A total of 2992 one-to-one orthogroups shared by the 13 Hymenoptera species were extracted for phylogenetic analysis. The protein sequences in each orthogroup were independently aligned by MAFFT v7 [21] and filtered by trimAl v1.2 [22] with the default parameters. These sequences were concatenated to generate a supergene sequence, which was used for tree construction. The phylogenetic tree was constructed by maximum likelihood (ML) using IQ-TREE v2.1.2 [23] with the best model (LG + I + G) estimated by ModelFinder [24] . Statistical support for the phylogenetic trees was assessed by Ultrafast bootstrap [25] analysis using 1000 replicates. The standard concatenation approaches do not model discordance among gene trees beyond differences in sequence evolution rates [144]. Many studies have shown that incomplete lineage sorting (ILS) has the potential to lead to incorrect topology, possibly due to the estimation bias in a concatenated analysis where the mixture of gene trees represents a model violation [145]. To overcome these limitations, ASTRAL-III [146], a multispecies coalescent tool, was used to summarize all the 2992 gene trees and measure branch supports as local posterior probabilities. Both concatenation and multispecies coalescent approach yield the same topology species tree. The MCMCtree program in the PAML package v4.9e [147] was used to estimate divergence time based on protein sequences. Five calibration time points based on a previous study were used, Orussoidea+Apocrita: 211-289 million years ago (mya), Apocrita: 203-276 mya, Ichneumonoidea: 151-218 mya, Chalcidoidea: 105-159 mya, Aculeata: 160-224 mya [2].

\section{Correlation between genome size and TE content}

Considering the phylogenetic relationships of the species studied, we first used the $\mathrm{R}$ caper package v1.0.1 (https://CRAN.R-project.org/package = caper) to test the 
effect of the phylogeny on the correlation between genome size and TE content. We obtained a lambda parameter from 0 to 1 ( 0 indicates covariances between taxa are negligible in these clades and thus those correlations are not biased by the phylogeny, and 1 means the evolutionary relationships among surveyed species induced a bias in the correlation calculation) by fitting a linear model on the data. We then computed corrected Pearson's correlation between the genome size and the TE content using the ape package v5.4-1 [148] in R if evolutionary relationships induced a bias in the correlation calculation.

\section{Identification of positively selected genes}

We used two packages, PAML v4.9 [147] and HyPhy [149] to detect positive selection signals on the G. flavifemur branch. In total, 2992 single-copy gene families were used for positive selection analyses. For PAML analysis, these single-copy genes were detected using the optimized branch-site model. A likelihood ratio test (LRT) was conducted to compare the null model (sites under neutrally and under purifying selection) and the alternative model (sites under positive selection on the foreground branch). The $\mathrm{p}$ values were computed based on chi-square statistics with a false discovery rate (FDR), and genes with $\mathrm{p}$-adjusted value less than 0.05 were identified as positive selection genes. For HyPhy analysis, the aBSREL algorithm [150] was used for positive selection signal searching. Genes with test $p$ values $<0.05$ were considered to be under positive selection. Finally, 183 genes detected by both methods were treated as candidates that underwent positive selection.

\section{Identification of rapidly evolving proteins}

Based on 2992 single-copy proteins, we used a rankbased branch length comparison method to identify rapidly evolving proteins on G. flavifemur branch as used in the Trichogramma genome [14]. In brief, a phylogenetic tree of each single-copy orthogroup was reconstructed using IQ-TREE v2.1.2 [23]. We then compared the rank of total branch length and the rank of G. flavifemur branch length. To avoid overrepresentation among any protein category based on general evolutionary rates, we next binned proteins into groups of 300 based on the total branch length rank. Rapidly evolving proteins were defined as proteins with the top $10 \%$ largest values of the G. flavifemur branch length rank minus the total branch length rank in each group.

\section{Gene family expansion and contraction}

We used CAFE v4.2.1 [151] to analysis the gene family expansion and contraction. The results from OrthoFinder and a phylogenetic tree with divergence times were used as inputs.

\section{Identification of venom gland-associated genes in $G$. flavifemur}

To identify the venom gland-associated genes, we firstly calculated the expression level of each transcript in the venom gland and the carcass (i.e., adult female tissues minus the venom gland) using RSEM v1.3.1 [124]. To qualify a gene as a venom gland-associated gene, it must be (a) among the top 500 expressed genes in the venom gland transcriptome, and (b) highly expressed in the venom gland relative to the carcass $(q<0.05)$, and (c) not highly expressed in the carcass (median FPKM $<50$ ). The signal peptide of each venom associate gene was predicted using the SignalP v5.0b [152].

\section{Identification of genes involved in detoxification and chemosensory}

To identify genes involved in detoxification and chemosensory, cytochrome P450s, glutathione S-transferases (GSTs), ATP-binding cassette transporters (ABCs), gustatory receptors (GRs), ionotropic receptors (IRs), olfactory receptors (ORs), odorant binding proteins (OBPs), sensory neuron membrane proteins (SNMPs), and chemosensory proteins (CSPs) protein sequences of wellannotated insects retrieved from Uniprot were used as queries to search against the predicted protein sequences from G. flavifemur and other 12 hymenopteran genomes mentioned in the comparative genomics section using BLASTP v2.8.1 [15] (-evalue 1e-5). All candidate detoxification and chemosensory genes were further checked for the presence of their characteristic domains to confirm their identity, P450s: PF00067, GSTs: PF00043 or PF02798, ABCs: PF00005, GRs: PF08395 or PF06151, IRs: PF00060, ORs: PF02949 or PF13853, OBPs: PF01395, SNMPs: PF01130, CSPs: PF03392.

\section{Chitinase-like genes}

To annotate the GH-18 chitinase-like genes, a number of well-annotated insect chitinase protein sequences were used as queries in a BLASTP search (-evalue 1e-5) against the proteins of 17 insect genomes including 13 hymenopteran genomes mentioned in the comparative genomics section, Bombyx mori (RefSeq accession: GCF_014905235.1), Tribolium castaneum (RefSeq accession: GCF_000002335.3), Drosophila melanogaster (RefSeq accession: GCF_000001215.4), and Acyrthosiphon pisum (RefSeq accession: GCF_005508785.1). We next checked the putative chitinase-like genes containing the glycosyl hydrolase 18 (GH-18) domain using hidden Markov models (HMM search) [153]. Finally, we manually confirmed the alignments of the GH18 domains and removed the sequences if they were only partially aligned. A HMM searching against the Pfam-A database [154] was then performed using the candidate chitinaselike proteins to identify additional domains (i.e., 
carbohydrate binding domains). Phylogenetic analysis of the chitinase-like proteins was performed using maximum likelihood methods with LG + R5 model (all chitinase-like proteins) and LG + G4 model (group 5 chitinase-like proteins) determined by ModelFinder [24] in IQ-TREE v2.1.2 [23]. Statistical support for all phylogenetic trees was assessed by Ultrafast bootstrap [25] analysis using 1000 replicates. Chitinase-like proteins were classified into subgroups based on the domain architecture and phylogenetic analysis as summarized in Arakane and Muthukrishnan [88]. The conserved catalytic domain in insect chitinase [86, 88, 155] was searched using the candidate chitinase-like proteins of G. flavifemur based on the multiple sequence alignment generated by MAFFT v 7.471 [21] with L-INS-I model.

\section{Neprilysin-like genes}

To identify neprilysin-like genes, we retrieved the wellannotated neprilysin sequences from UniProtKB/SwissProt as queries. BLASTP (-evalue 1e-5) was performed for protein searching in the 17 insect genomes described in chitinase-like gene section. Each protein sequence obtained was subsequently used for searching against Pfam-A [154] database by HMMscan v3.3.2 [153] (--cut_ ga) to confirm the presence of the Peptidase_M13 domain. Candidate neprilysin-like proteins were manually checked for the alignments of Peptidase_M13 domain. To gain insights into the venom neprilysin-like genes of other hymenopteran species, we annotated the neprilysin-like genes in the venom genes of additional 30 hymenopteran insects (15 in Parasitoida and 15 in Aculeate) using the same method $[35,67-70,75,77,89,90$, 156-164]. The phylogeny of the hymenopterans in Fig. $5 \mathrm{c}$ was obtained from previous studies $[2,91]$. Phylogenetic analysis of the neprilysin-like genes was performed using maximum likelihood methods using LG + R9 model determined by ModelFinder [24] in IQ-TREE v2.1.2 [23]. Statistical support for all phylogenetic trees was assessed by Ultrafast bootstrap [25] analysis using 1000 replicates.

\section{Identification of opsin genes}

Amino acid sequences of opsin genes in A. mellifera, $D$. melanogaster, and $T$. castaneum were obtained from UniProt. They were used for searching the proteins against 13 hymenopteran genomes using BLASTP v2.8.1 [16] (-evalue 1e-10). To discriminate opsins from other G-protein-coupled receptors (GPCRs), we used a combination of sequence similarity and motif analysis described in Feuda et al. [98]. Briefly, an opsin gene should have a top BLASTP hit with opsin in Uniprot and/or contain a recognizable retinal-binding domain.

\section{Enrichment analysis}

GO enrichment analyses were conducted by GOATOOLS v1.0.6 [165], a python library for gene ontology analysis.

\section{Abbreviations}

IDGF: Imaginal disc growth factor; LWS: Long-wave sensitive; GSTs: Glutathione S-transferases; ABCs: ATP-binding cassette transporters; GRs: Gustatory receptors; IRs: Ionotropic receptors; ORs: Olfactory receptors; OBPs: Odorant binding proteins; SNMPs: Sensory neuron membrane proteins; CSPs: Chemosensory proteins; BUSCO: Benchmarking universal single-copy orthologs; NCBI: National Center for Biotechnology Information;

TEs: Transposable elements; LTRs: Long terminal repeat retrotransposons; LINEs: Long interspersed nuclear elements; SINEs: Short interspersed nuclear elements; MITEs: Miniature inverted-repeat transposable elements; GO: Gene Ontology; FDR: False discovery rate; NDUFB3: NADH dehydrogenase ubiquinone 1 beta subcomplex subunit 3; NDUFA9: NADH dehydrogenase ubiquinone 1 alpha subcomplex subunit 9; mtTFB1: Mitochondrial transcription factor B1; PTCD3: Pentatricopeptide repeat domain-containing protein 3; TLN1: Talin-1; TBCE: Tubulin-specific chaperone E; Scgß: Betasarcoglycan; SPG11: Spatacsin 11; ALS2: Amyotrophic lateral sclerosis 2; TWF1: Twinfilin 1; SIM: Single-minded; AGO3: Argonaute 3; ETFRF1: Electron transfer flavoprotein regulatory factor 1; NDUFB7: NADH dehydrogenase ubiquinone 1 beta subcomplex subunit 7; cGK: cGMP-dependent protein kinase; GTF2IRD: General transcription factor II-I repeat domain-containing; VGGs: Venom gland-associated genes; FPKM: Fragments per kilobase per million mapped fragments; GH18: Glycoside hydrolase 18; UV: Ultraviolet; SDS: Sodium dodecyl sulfate; KEGG: Kyoto Encyclopedia of Genes and Genomes; Mya: Million years ago; LRT: Likelihood ratio test; HMM: Hidden Markov models

\section{Supplementary Information}

The online version contains supplementary material available at https://doi. org/10.1186/s12915-021-01081-6.

Additional file 1: Supplementary Table 1. Statistics of Nanopore sequencing. Supplementary Table 2. Statistics of Illumina sequencing. Supplementary Table 3. Statistics of Gonatopus flavifemur genome assembly by Nanopore reads. Supplementary Table 4. Statistics of Gonatopus flavifemur genome assembly after error-corrected by Illumina and Nanopore reads. Supplementary Table 5. Genome size of G. flavifemur estimated by flow cytometry and 17-mer analysis. Supplementary Table 6. The mapping rate with short sequencing reads of Gonatopus flavifemur genome. Supplementary Table 7. Genes in detoxification system and chemoreception system in 13 hymenopterans. Supplementary Table 8. TE sequences in Gonatopus flavifemur and other surveyed species. Supplementary Table 9. Characteristics of DNA TEs in Gonatopus flavifemur. Supplementary Table 10. GO enrichment analysis of Aculeate-specific genes, Biological Process category (padj < 0.05 ). Supplementary Table 11. GO enrichment analysis of Gonatopus flavifemur special genes. (padj < 0.05). Supplementary Table 12. Genes under positive selection in Gonatopus flavifemur detected by both aBSREL model in HyPhy and branch-site model in PAML. Supplementary Table 13. $\mathrm{GO}$ enrichment analysis of Gonatopus flavifemur expanded gene families, Biological Process category (padj <0.05). Supplementary Table 14. Venom gland-associated genes (VGGs) in Gonatopus flavifemur. Supplementary Table 15. GO enrichment analysis of VGGs in Gonatopus flavifemur, Biological Process category (padj < 0.05). Supplementary Table 16. Chitinase-like genes in Gonatopus flavifemur and other species. Supplementary Table 17. Neprilysin-like genes in Gonatopus flavifemur and other Hymenoptera genomes. Supplementary Table 18. Upregulated genes in the prey-feeding female transcriptomes compared to the sucrose-feeding female transcriptomes. Supplementary Table 19. Yellow genes and expression level in Gonatopus flavifemur. Supplementary Table 20. Downregulated genes in the prey-feeding female transcriptomes compared to the sucrose-feeding female transcriptomes. Supplementary Table 21. GO enrichment analysis of extremely female-biased genes in Gonatopus flavifemur. Biological Process category (padj < 0.05). 
Supplementary Table 22. GO enrichment analysis of extremely malebiased genes in Gonatopus flavifemur. Biological Process category (padj < 0.05). Supplementary Table 23. Extremely male-biased genes in Gonatopus flavifemur. Supplementary Table 24. Sample information for sequencing used in this study.

Additional file 2: Supplementary Figure 1. Genome size of $G$. flavifemur estimated by k-mer analysis. Supplementary Figure 2. Flow cytometry histograms of Drosophila melanogaster (A) and G. flavifemur (B). Supplementary Figure 3. Concatenated-and ASTRAL-based phylogenetic trees. Supplementary Figure 4. Rank-based branch length comparison of protein evolution rates in the G. flavifemur branch.

Supplementary Figure 5. Venom gland-associated genes (VGGs) in G. flavifemur. Supplementary Figure 6. Multiple sequence alignment of the active site motif (DxxDxDxE) of IDGFs in G. flavifemur and other 12 hymenopterans.

Additional file 3. Bacterial contaminating contigs in the G. flavifemur genome assembly.

\section{Acknowledgements}

We thank Dr. Qiang Fu working at China Rice Research Institute for his help on collecting the dryinid wasp G. flavifemur from rice fields, and Dr Kang He working at Zhejiang University for his help on the experiment of flow cytometry. We thank the help from Nextomics Biosciences Institute for technical support in Nanopore and Illumina sequencing. Specially, we would like to express our thanks to Prof. Zaifu Xu from South China Agricultural University, who was a pioneer in the research of Chinese Dryinidae and passed away in 2017.

\section{Authors' contributions}

G.Y.Y. and X.H.Y. conceived and designed the whole project. H.M.X., F.L., and Q.F coordinated the project. C.D., R.H., and S.X. prepared the samples for Nanopore and Illumina sequencing. Y.Y., X.H.Y., and Y.M. annotated the genome. X.H.Y. and Y.Y. conducted the phylogenetic, comparative genomics, and gene family evolution analyses. Y.Y., X.H.Y., and L.X. analyzed genome size evolution. Y.Y., X.H.Y., and Y.M. conducted gene family annotation. X.H.Y. and Y.Y. performed venom gland-associated gene analysis. Y.Y., X.H.Y., and Y.S.C. conducted the transcriptome analysis. X.H.Y. and Y.Y. prepared figures, tables, and supporting information. X.H.Y. and Y.Y. wrote the draft manuscript. Y.H.S., L.X., F.L., Q.F., H.M.X., and G.Y.Y. improved and revised the manuscript. All authors read and approved the final manuscript.

\section{Funding}

This work was supported by Key Program of National Natural Science Foundation of China (NSFC) (Grant no. 31830074 to G.Y.Y.), Major International (Regional) Joint Research Project of NSFC (Grant no. 31620103915 to G.Y.Y.), Program for Chinese Innovation Team in Key Areas of Science and Technology of Ministry of Science and Technology of the People's Republic of China (Grant/Award Number: 2016RA4008), and Program for Chinese Outstanding Talents in Agricultural Scientific Research of the Ministry of Agriculture and Rural Affairs of the People's Republic of China. X.H.Y. and L.X. thanks "Academic Star" Program for Ph. D Student of Zhejiang University for support.

\section{Availability of data and materials}

All the data generated in this study are available at the National Center for Biotechnology Information (NCBI), under BioProject number PRJNA695321 [166]. The genome assembly has been deposited at GenBank under accession JAFFJZ000000000 [167]. The genome and transcriptome sequencing data have been deposited in the NCBI Sequence Read Archive (SRA) database (SRR14374062, SRR14374063 and SRR13625245-SRR13625267). In addition, all the sequencing data and annotation information are available in InsectBase (http://insect-genome.com/Gfla/) [168].

\section{Declarations}

Ethics approval and consent to participate

Not applicable.

\section{Consent for publication}

Not applicable.

\section{Competing interests}

The authors declare that they have no conflict of interest.

\section{Author details}

'State Key Laboratory of Rice Biology and Ministry of Agricultural and Rural Affairs Key Laboratory of Molecular Biology of Crop Pathogens and Insects, Zhejiang University, Hangzhou, China. ${ }^{2}$ Department of Biology, University of Rochester, Rochester, NY, USA. ${ }^{3}$ Key Laboratory of Crop Growth and Development Regulation of Jiangxi Province, College of Life Sciences and Resource Environment, Yichun University, Yichun, China.

Received: 14 February 2021 Accepted: 30 June 2021

Published online: 27 July 2021

\section{References}

1. Branstetter MG, Danforth BN, Pitts JP, Faircloth BC, Ward PS, Buffington ML, et al. Phylogenomic insights into the evolution of stinging wasps and the origins of ants and bees. Curr Biol. 2017;27(7):1019-25. https://doi.org/10.101 6/j.cub.2017.03.027.

2. Peters RS, Krogmann L, Mayer C, Donath A, Gunkel S, Meusemann K, et al. Evolutionary history of the Hymenoptera. Curr Biol. 2017;27(7):1013-8. https://doi.org/10.1016/j.cub.2017.01.027.

3. Olmi M. A revision of the Dryinidae (Hymenoptera). Memoirs Am Entomol Inst. 1984;37:947-1913.

4. Guglielmino A. Dryinidae (Hymenoptera Chrysidoidea): an interesting group among the natural enemies of the Auchenorrhyncha (Hemiptera). Denisia. 2002;4:549-56.

5. Melo GA, Marcel MG, Garcete-Barrett BR. Origin and occurrence of predation among Hymenoptera: a phylogenetic perspective. In: Polidori $C$ editor. Predation in the Hymenoptera: an evolutionary perspective. Kerala: Transworld Research Network; 2011. p. 1-22.

6. Xu Z, Olmi M, He J. Dryinidae of the oriental region (Hymenoptera: Chrysidoidea). Zootaxa. 2013;3614(1):1-460. https://doi.org/10.11646/zoota xa.3614.1.1.

7. Tian JC, Romeis J, Liu K, Zhang FC, Zheng XS, Xu HX, et al. Assessing the effects of Cry $1 \mathrm{C}$ rice and Cry2A rice to Pseudogonatopus flavifemur, a parasitoid of rice planthoppers. Sci Rep. 2017;7(1):7838. https://doi.org/10.1 038/s41598-017-08173-w.

8. He J, He Y, Lai F, Chen X, Fu Q. Biological traits of the pincer wasp Gonatopus flavifemur (Esaki \&amp; Hashimoto) associated with different stages of its host, the brown planthopper, Nilaparvata lugens (Stål). Insects. 2020;11(5):279. https://doi.org/10.3390/insects11050279.

9. Mora-Kepfer F, Espinoza AM. Parasitism and predation of the planthopper Tagosodes orizicolus (Homoptera: Delphacidae) by a dryinid parasitoid in Costa Rica. Rev Biol Trop. 2009;57(Suppl 1):203-11.

10. Uy FMK, Espinoza AM. Differential host handling behavior between feeding and oviposition in the parasitic wasp Haplogonatopus hernandezae. J Insect Behav. 2018;31(5):569-84. https://doi.org/10.1007/s10905-018-9699-4.

11. Wheeler D, Redding AJ, Werren JH. Characterization of an ancient Lepidopteran lateral gene transfer. PLOS One. 2013;8(3):e59262. https://doi. org/10.1371/journal.pone.0059262.

12. Poynton HC, Hasenbein S, Benoit JB, Sepulveda MS, Poelchau MF, Hughes DST, et al. The toxicogenome of Hyalella azteca: a model for sediment ecotoxicology and evolutionary toxicology. Environ Sci Technol. 2018;52(10): 6009-22. https://doi.org/10.1021/acs.est.8b00837.

13. Ye XH, Yan ZC, Yang Y, Xiao S, Chen LF, Wang JL, et al. A chromosome-level genome assembly of the parasitoid wasp Pteromalus puparum. Mol Ecol Resour. 2020;20(5):1384-402. https://doi.org/10.1111/1755-0998.13206.

14. Lindsey ARI, Kelkar YD, Wu X, Sun D, Martinson EO, Yan Z, et al. Comparative genomics of the miniature wasp and pest control agent Trichogramma pretiosum. BMC Biol. 2018;16(1):54. https://doi.org/10.1186/ s12915-018-0520-9.

15. Olafson PU, Aksoy S, Attardo GM, Buckmeier G, Chen X, Coates CJ, et al. The genome of the stable fly, Stomoxys calcitrans, reveals potential mechanisms underlying reproduction, host interactions, and novel targets for pest control. BMC Biol. 2021;19(1):41. https://doi.org/10.1186/s12915-021-00975-9.

16. Camacho C, Coulouris G, Avagyan V, Ma N, Papadopoulos J, Bealer K, et al. BLAST+: architecture and applications. BMC Bioinformatics. 2009;10(1):421. https://doi.org/10.1186/1471-2105-10-421.

17. Overbeek R, Olson R, Pusch GD, Olsen GJ, Davis JJ, Disz T, et al. The SEED and the rapid annotation of microbial genomes using subsystems 
technology (RAST). Nucleic Acids Res. 2014;42(D1):D206-14. https://doi.org/1 $0.1093 / \mathrm{nar} / \mathrm{gkt} 1226$

18. Huerta-Cepas J, Forslund K, Coelho LP, Szklarczyk D, Jensen LJ, von Mering C, et al. Fast genome-wide functional annotation through orthology assignment by eggNOG-Mapper. Mol Biol Evol. 2017;34(8):2115-22. https:// doi.org/10.1093/molbev/msx148.

19. Cabanettes F, Klopp C. D-GENIES: dot plot large genomes in an interactive, efficient and simple way. PeerJ. 2018;6:e4958. https://doi.org/10.7717/peerj.4958.

20. Emms DM, Kelly S. OrthoFinder: phylogenetic orthology inference for comparative genomics. Genome Biol. 2019;20(1):238. https://doi.org/10.11 86/s13059-019-1832-y.

21. Katoh K, Standley DM. MAFFT multiple sequence alignment software version 7: improvements in performance and usability. Mol Biol Evol. 2013; 30(4):772-80. https://doi.org/10.1093/molbev/mst010.

22. Capella-Gutiérrez S, Silla-Martínez JM, Gabaldón T. trimAl: a tool for automated alignment trimming in large-scale phylogenetic analyses. Bioinformatics. 2009; 25(15):1972-3. https://doi.org/10.1093/bioinformatics/btp348.

23. Minh BQ, Schmidt HA, Chernomor O, Schrempf D, Woodhams MD, von Haeseler A, et al. IQ-TREE 2: New models and efficient methods for phylogenetic inference in the genomic era. Mol Biol Evol. 2020;37(5):1530-4. https://doi.org/10.1093/molbev/msaa015.

24. Kalyaanamoorthy S, Minh BQ, Wong TKF, von Haeseler A, Jermiin LS. ModelFinder: fast model selection for accurate phylogenetic estimates. Nat Methods. 2017;14(6):587-9. https://doi.org/10.1038/nmeth.4285.

25. Hoang DT, Chernomor O, von Haeseler A, Minh BQ, Vinh LS. UFBoot2: Improving the ultrafast bootstrap approximation. Mol Biol Evol. 2018;35(2): 518-22. https://doi.org/10.1093/molbev/msx281.

26. Darby AC, Choi JH, Wilkes T, Hughes MA, Werren JH, Hurst GDD, et al. Characteristics of the genome of Arsenophonus nasoniae, son-killer bacterium of the wasp Nasonia. Insect Mol Biol. 2010;19:75-89. https://doi. org/10.1111/j.1365-2583.2009.00950.x.

27. Wallberg A, Bunikis I, Pettersson OV, Mosbech MB, Childers AK, Evans JD, et al. A hybrid de novo genome assembly of the honeybee, Apis mellifera, with chromosome-length scaffolds. BMC Genomics. 2019;20(1):275. https:// doi.org/10.1186/s12864-019-5642-0.

28. Gokhman VE. Karyotypes of parasitic Hymenoptera. Dordrecht: Springer Netherlands; 2009. https://doi.org/10.1007/978-1-4020-9807-9.

29. Branstetter M, Childers AK, Cox-Foster D, Hopper KR, Kapheim KM, Toth AL, et al. Genomes of the Hymenoptera. Curr Opin Insect Sci. 2018;25:65-75. https://doi.org/10.1016/j.cois.2017.11.008.

30. Petersen M, Armisén D, Gibbs RA, Hering L, Khila A, Mayer G, et al. Diversity and evolution of the transposable element repertoire in arthropods with particular reference to insects. BMC Evol Biol. 2019;19(1):11. https://doi.org/1 0.1186/s12862-018-1324-9.

31. Brand P, Saleh N, Pan H, Li C, Kapheim KM, Ramírez SR. The nuclear and mitochondrial genomes of the facultatively eusocial orchid Bee Euglossa dilemma. G3 (Bethesda). 2017;7:2891-8.

32. Wang $X$, Fang $X$, Yang $P$, Jiang $X$, Jiang F, Zhao $D$, et al. The locust genome provides insight into swarm formation and long-distance flight. Nat Commun. 2014;5(1):2957. https://doi.org/10.1038/ncomms3957.

33. Sun C, Huang J, Wang Y, Zhao X, Su L, Thomas GWC, et al. Genus-wide characterization of bumblebee genomes provides insights into their evolution and variation in ecological and behavioral traits. Mol Biol Evol. 2020;38:486-501.

34. Wu C, Lu J. Diversification of transposable elements in arthropods and its impact on genome evolution. Genes. 2019;10(5):338. https://doi.org/10.33 90/genes10050338.

35. Oeyen JP, Baa-Puyoulet P, Benoit JB, Beukeboom LW, Bornberg-Bauer $E_{\text {, }}$ Buttstedt $A$, et al. Sawfly genomes reveal evolutionary acquisitions that fostered the mega-radiation of parasitoid and eusocial Hymenoptera. Genome Biol Evol. 2020;12(7):1099-188. https://doi.org/10.1093/gbe/evaa106.

36. Kraaijeveld K, Neleman P, Mariën J, de Meijer E, Ellers J. Genomic resources for Goniozus legneri, Aleochara bilineata and Paykullia maculata, representing three independent origins of the parasitoid lifestyle in insects. G3 (Bethesda). 2019;9:987-91.

37. Rhooms S-K, Murari A, Goparaju NS, Vilanueva M, Owusu-Ansah E. Insights from Drosophila on mitochondrial complex I. Cell Mol Life Sci. 2020;77(4): 607-18. https://doi.org/10.1007/s00018-019-03293-0.

38. Matsushima Y, Adán C, Garesse R, Kaguni LS. Drosophila mitochondrial transcription factor B1 modulates mitochondrial translation but not transcription or DNA copy number in Schneider cells. J Biol Chem. 2005: 280(17):16815-20. https://doi.org/10.1074/jbc.M500569200.
39. Grover R, Burse SA, Shankrit S, Aggarwal A, Kirty K, Narta K, et al. Myg1 exonuclease couples the nuclear and mitochondrial translational programs through RNA processing. Nucleic Acids Res. 2019;47(11):5852-66. https://doi. org/10.1093/nar/gkz371.

40. Davies SMK, Rackham O, Shearwood A-MJ, Hamilton KL, Narsai R, Whelan J, et al. Pentatricopeptide repeat domain protein 3 associates with the mitochondrial small ribosomal subunit and regulates translation. FEBS Lett. 2009;583(12):1853-8. https://doi.org/10.1016/j.febslet.2009.04.048.

41. Senetar MA, McCann RO. Gene duplication and functional divergence during evolution of the cytoskeletal linker protein talin. Gene. 2005;362:14152. https://doi.org/10.1016/j.gene.2005.08.012.

42. Jin S, Pan L, Liu Z, Wang Q, Xu Z, Zhang YQ. Drosophila Tubulin-specific chaperone $E$ functions at neuromuscular synapses and is required for microtubule network formation. Development. 2009;136(9):1571-81. https:// doi.org/10.1242/dev.029983.

43. Hashimoto R, Yamaguchi M. Genetic link between $\beta$-sarcoglycan and the Egfr signaling pathway. Biochem Biophys Res Commun. 2006;348(1):212-21. https://doi.org/10.1016/j.bbrc.2006.07.045.

44. Pérez-Brangulí F, Mishra HK, Prots I, Havlicek S, Kohl Z, Saul D, et al. Dysfunction of spatacsin leads to axonal pathology in SPG17-linked hereditary spastic paraplegia. Hum Mol Genet. 2014;23(18):4859-74. https:// doi.org/10.1093/hmg/ddu200.

45. Takayama Y, Itoh RE, Tsuyama T, Uemura T. Age-dependent deterioration of locomotion in Drosophila melanogaster deficient in the homologue of amyotrophic lateral sclerosis 2. Genes Cells Devoted Mol Cell Mech. 2014; 19(6):464-77. https://doi.org/10.1111/gtc.12146.

46. Wang D, Zhang L, Zhao G, Wahlström G, Heino TI, Chen J, et al. Drosophila twinfilin is required for cell migration and synaptic endocytosis. J Cell Sci. 2010;123(9):1546-56. https://doi.org/10.1242/jcs.060251.

47. Wahlström G, Vartiainen M, Yamamoto L, Mattila PK, Lappalainen P, Heino TI. Twinfilin is required for actin-dependent developmental processes in Drosophila. J Cell Biol. 2001;155(5):787-96. https:/doi.org/10.1083/jcb.200108022.

48. Nambu JR, Lewis JO, Wharton KA, Crews ST. The Drosophila single-minded gene encodes a helix-loop-helix protein that acts as a master regulator of CNS midline development. Cell. 1991;67(6):1157-67. https://doi.org/10.1016/ 0092-8674(91)90292-7.

49. Sarot E, Payen-Groschêne $G$, Bucheton A, Pélisson A. Evidence for a piwidependent RNA silencing of the gypsy endogenous retrovirus by the Drosophila melanogaster flamenco gene. Genetics. 2004;166(3):1313-21. https://doi.org/10.1534/genetics.166.3.1313.

50. Thomson T, Lin H. The biogenesis and function PIWI proteins and piRNAs: progress and prospect. Annu Rev Cell Dev Biol. 2009;25(1):355-76. https:// doi.org/10.1146/annurev.cellbio.24.110707.175327.

51. Ku HY, Lin H. PIWI proteins and their interactors in piRNA biogenesis, germline development and gene expression. Natl Sci Rev. 2014;1 (2):205-18. https://doi.org/10.1093/nsr/nwu014.

52. Sun $\mathrm{YH}$, Xie LH, Zhuo X, Chen Q, Ghoneim D, Zhang B, et al. Domestic chickens activate a piRNA defense against avian leukosis virus. eLife. 2017;6: e24695. https://doi.org/10.7554/eLife.24695.

53. Floyd BJ, Wilkerson EM, Veling MT, Minogue CE, Xia C, Beebe ET, et al. Mitochondrial protein interaction mapping identifies new regulators of respiratory chain function. Mol Cell. 2016;63(4):621-32. https://doi.org/10.101 6/j.molcel.2016.06.033.

54. Shao J, Welch WJ, DiProspero NA, Diamond MI. Phosphorylation of profilin by ROCK1 regulates polyglutamine aggregation. Mol Cell Biol. 2008;28(17): 5196-208. https://doi.org/10.1128/MCB.00079-08.

55. MacPherson MR, Lohmann SM, Davies SA. Analysis of Drosophila cGMPdependent protein kinases and assessment of their in vivo roles by targeted expression in a renal transporting epithelium. J Biol Chem. 2004;279(38): 40026-34. https://doi.org/10.1074/jbc.M405619200.

56. de la Cruz IP, Levin JZ, Cummins C, Anderson P, Horvitz HR. sup-9, sup10 , and unc-93 may encode components of a two-pore $\mathrm{K}+$ channel that coordinates muscle contraction in Caenorhabditis elegans. J Neurosci. 2003;23(27):9133-45. https://doi.org/10.1523/JNEUROSCI.23-2709133.2003

57. Tussié-Luna MI, Bayarsaihan D, Ruddle FH, Roy AL. Repression of TFII-Idependent transcription by nuclear exclusion. Proc Natl Acad Sci U S A. 2001;98(14):7789-94. https://doi.org/10.1073/pnas.141222298.

58. Martinson EO, Wheeler D, Wright J, Mrinalini, Siebert AL, Werren JH. Nasonia vitripennis venom causes targeted gene expression changes in its fly host. Mol Ecol. 2014;23(23):5918-30. https://doi.org/10.1111/mec.12967. 
59. Mrinalini, Siebert AL, Wright J, Martinson E, Wheeler D, Werren JH. Parasitoid venom induces metabolic cascades in fly hosts. Metabolomics. 2015;11:350-66

60. Rivers DB, Denlinger DL. Redirection of metabolism in the flesh fly, Sarcophaga bullata, following envenomation by the ectoparasitoid Nasonia vitripennis and correlation of metabolic effects with the diapause status of the host. J Insect Physiol. 1994;40(3):207-15. https://doi.org/10.1016/0022-1 910(94)90044-2

61. Martinson EO, Mrinalini, Kelkar YD, Chang CH, Werren $\mathrm{JH}$. The evolution of venom by co-option of single-copy genes. Curr Biol. 2017;27(13):2007-13. https://doi.org/10.1016/j.cub.2017.05.032.

62. Moreau SJM, Asgari S. Venom proteins from parasitoid wasps and their biological functions. Toxins. 2015;7(7):2385-412. https://doi.org/10.3390/ toxins 7072385.

63. Casewell NR, Wüster W, Vonk FJ, Harrison RA, Fry BG. Complex cocktails: the evolutionary novelty of venoms. Trends Ecol Evol. 2013;28(4):219-29. https:// doi.org/10.1016/j.tree.2012.10.020.

64. Walker AA, Robinson SD, Yeates DK, Jin J, Baumann K, Dobson J, et al. Entomo-venomics: The evolution, biology and biochemistry of insect venoms. Toxicon. 2018;154:15-27. https://doi.org/10.1016/j.toxicon.2018.09. 004.

65. Kote S, Faktor J, Dapic I, Mayordomo MY, Kocikowski M, Kagansky A, et al. Analysis of venom sac constituents from the solitary, aculeate wasp Cerceris rybyensis. Toxicon. 2019;169:1-4. https://doi.org/10.1016/j.toxicon.2019.07. 012.

66. Robinson SD, Mueller A, Clayton D, Starobova H, Hamilton BR, Payne RJ, et al. A comprehensive portrait of the venom of the giant red bull ant, Myrmecia gulosa, reveals a hyperdiverse hymenopteran toxin gene family. Sci Adv. 2018:4:eaau4640

67. de Graaf DC, Aerts M, Brunain M, Desjardins CA, Jacobs FJ, Werren JH, et al. Insights into the venom composition of the ectoparasitoid wasp Nasonia vitripennis from bioinformatic and proteomic studies. Insect Mol Biol. 2010; 19(Suppl 1):11-26. https://doi.org/10.1111/j.1365-2583.2009.00914.x.

68. Yan Z, Fang Q, Wang L, Liu J, Zhu Y, Wang F, et al. Insights into the venom composition and evolution of an endoparasitoid wasp by combining proteomic and transcriptomic analyses. Sci Rep. 2016;6(1):19604. https://doi. org/10.1038/srep19604.

69. Yang L, Yang Y, Liu MM, Yan ZC, Qiu LM, Fang Q, et al. Identification and comparative analysis of venom proteins in a pupal ectoparasitoid, Pachycrepoideus vindemmiae. Front Physiol. 2020;11:9. https://doi.org/10.33 89/fphys.2020.00009.

70. Yoon KA, Kim K, Kim WJ, Bang WY, Ahn NH, Bae CH, et al. Characterization of venom components and their phylogenetic properties in some Aculeate bumblebees and wasps. Toxins. 2020;12(1):47. https://doi.org/10.3390/ toxins12010047.

71. Wan B, Poirie M, Gatti JL. Parasitoid wasp venom vesicles (venosomes) enter Drosophila melanogaster lamellocytes through a flotillin/lipid raft-dependent endocytic pathway. Virulence. 2020;11(1):1512-21. https://doi.org/10.1080/21 505594.2020.1838116.

72. Martinson EO, Martinson VG, Edwards R, Mrinalini M, Werren JH. Laterally transferred gene recruited as a venom in parasitoid wasps. Mol Biol Evol. 2016;33(4):1042-52. https://doi.org/10.1093/molbev/msv348.

73. Sitnik JL, Francis C, Hens K, Huybrechts R, Wolfner MF, Callaerts P. Neprilysins: An evolutionarily conserved family of metalloproteases that play important roles in reproduction in Drosophila. Genetics. 2014;196(3):781-97. https://doi.org/10.1534/genetics.113.160945.

74. Liu G, Zhou Y, Liu D, Wang Q, Ruan Z, He Q, et al. Global transcriptome analysis of the tentacle of the jellyfish Cyanea capillata using deep sequencing and expressed sequence tags: insight into the toxin- and degenerative disease-related transcripts. PLoS One. 2015;10(11):e0142680. https://doi.org/10.1371/journal.pone.0142680.

75. Arvidson R, Kaiser M, Pan S, Libersat F, Adams ME. Bioinformatic and functional analysis of venom from the jewel wasp Ampulex compressa. FASEB J. 2016;30:819.1

76. Tan KY, Tan $\mathrm{CH}$, Chanhome L, Tan NH. Comparative venom gland transcriptomics of Naja kaouthia (monocled cobra) from Malaysia and Thailand: elucidating geographical venom variation and insights into sequence novelty. PeerJ. 2017;5:e3142. https://doi.org/10.7717/peerj.3142.

77. Arvidson R, Kaiser M, Lee SS, Urenda JP, Dail C, Mohammed H, et al. Parasitoid jewel wasp mounts multipronged neurochemical attack to hijack a host brain. Mol Cell Proteomics. 2019;18(1):99-114. https://doi.org/10.1 074/mcp.RA118.000908.
78. Zobel-Thropp PA, Mullins J, Kristensen C, Kronmiller BA, David CL, Breci LA, et al. Not so dangerous after all? Venom composition and potency of the pholcid (daddy long-leg) spider Physocyclus mexicanus. Front Ecol Evol. 2019;7:256. https://doi.org/10.3389/fevo.2019.00256.

79. Turner AJ, Isaac RE, Coates D. The neprilysin (NEP) family of zinc metalloendopeptidases: genomics and function. Bioessays. 2001;23(3):261-9. https://doi.org/10.1002/1521-1878(200103)23:3<261::AID-BIES1036>3.0.CO;2-K.

80. Ellsworth SA, Nystrom GS, Ward MJ, Freitas de Sousa LA, Hogan MP, Rokyta DR. Convergent recruitment of adamalysin-like metalloproteases in the venom of the red bark centipede (Scolopocryptops sexspinosus). Toxicon. 2019;168:1-15.

81. Park SY, Kim CH, Jeong WH, Lee JH, Seo SJ, Han YS, et al. Effects of two hemolymph proteins on humoral defense reactions in the wax moth, Galleria mellonella. Dev Comp Immunol. 2005;29(1):43-51. https://doi.org/1 0.1016/j.dci.2004.06.001.

82. Xue J, Zhou X, Zhang CX, Yu LL, Fan HW, Wang Z, et al. Genomes of the rice pest brown planthopper and its endosymbionts reveal complex complementary contributions for host adaptation. Genome Biol. 2014; 15(12):521. https://doi.org/10.1186/s13059-014-0521-0.

83. Xi Y, Pan PL, Ye YX, Yu B, Xu HJ, Zhang CX. Chitinase-like gene family in the brown planthopper, Nilaparvata lugens. Insect Mol Biol. 2015;24(1):29-40. https://doi.org/10.1111/imb.12133.

84. Nakabachi A, Shigenobu S, Miyagishima S. Chitinase-like proteins encoded in the genome of the pea aphid, Acyrthosiphon pisum. Insect Mol Biol. 2010; 19(Suppl 2):175-85. https://doi.org/10.1111/j.1365-2583.2009.00985.x.

85. Zhang J, Zhang X, Arakane Y, Muthukrishnan S, Kramer KJ, Ma E, et al. Comparative genomic analysis of chitinase and chitinase-like genes in the African malaria mosquito (Anopheles gambiae). PLOS One. 2011;6(5):e19899. https://doi.org/10.1371/journal.pone.0019899.

86. Zhu Q, Deng Y, Vanka P, Brown SJ, Muthukrishnan S, Kramer KJ. Computational identification of novel chitinase-like proteins in the Drosophila melanogaster genome. Bioinformatics. 2004;20(2):161-9. https:// doi.org/10.1093/bioinformatics/bth020.

87. Pan $Y, L u ̈ P$, Wang $Y$, Yin $L, M a ~ H, M a ~ G$, et al. In silico identification of novel chitinase-like proteins in the silkworm, Bombyx mori, genome. J Insect Sci. 2012;12:150.

88. Arakane $Y$, Muthukrishnan S. Insect chitinase and chitinase-like proteins. Cell Mol Life Sci. 2010;67(2):201-16. https://doi.org/10.1007/s00018-009-0161-9.

89. Vincent B, Kaeslin M, Roth T, Heller M, Poulain J, Cousserans F, et al. The venom composition of the parasitic wasp Chelonus inanitus resolved by combined expressed sequence tags analysis and proteomic approach. BMC Genomics. 2010;11(1):693. https://doi.org/10.1186/1471-2164-11-693.

90. Lin Z, Wang RJ, Cheng Y, Du J, Volovych O, Han LB, et al. Insights into the venom protein components of Microplitis mediator, an endoparasitoid wasp. Insect Biochem Mol Biol. 2019;105:33-42. https://doi.org/10.1016/j.ibmb.201 8.12.013.

91. Chen XX, van Achterberg C. Systematics, phylogeny, and evolution of Braconid wasps: 30 years of progress. Annu Rev Entomol. 2019;64(1):335-58. https://doi.org/10.1146/annurev-ento-011118-111856.

92. Roy SG, Raikhel AS. Nutritional and hormonal regulation of the TOR effector 4E-binding protein (4E-BP) in the mosquito Aedes aegypti. Faseb J. 2012; 26(3):1334-42. https://doi.org/10.1096/fj.11-189969.

93. Ferguson LC, Green J, Surridge A, Jiggins CD. Evolution of the insect yellow gene family. Mol Biol Evol. 2011;28(1):257-72. https://doi.org/10.1093/ molbev/msq192.

94. Drapeau MD, Albert S, Kucharski R, Prusko C, Maleszka R. Evolution of the Yellow/Major Royal Jelly Protein family and the emergence of social behavior in honey bees. Genome Res. 2006;16(11):1385-94. https://doi.org/1 $0.1101 /$ gr.5012006

95. Claycomb JM, Benasutti M, Bosco G, Fenger DD, Orr-Weaver TL. Gene amplification as a developmental strategy: isolation of two developmental amplicons in Drosophila. Dev Cell. 2004;6(1):145-55. https://doi.org/10.1016/ S1534-5807(03)00398-8.

96. Noh MY, Kim SH, Gorman MJ, Kramer KJ, Muthukrishnan S, Arakane Y. Yellow-g and Yellow-g2 proteins are required for egg desiccation resistance and temporal pigmentation in the Asian tiger mosquito, Aedes albopictus. Insect Biochem Mol Biol. 2020;122:103386. https://doi.org/10.1016/j.ibmb.202 0.103386 .

97. Xia AH, Zhou QX, Yu LL, Li WG, Yi YZ, Zhang YZ, et al. Identification and analysis of YELLOW protein family genes in the silkworm, Bombyx mori. BMC Genomics. 2006;7(1):195. https://doi.org/10.1186/1471-2164-7-195. 
98. Feuda R, Marlétaz F, Bentley MA, Holland PWH. Conservation, duplication, and divergence of five opsin genes in insect evolution. Genome Biol Evol. 2016;8(3):579-87. https://doi.org/10.1093/gbe/evw015.

99. Merzendorfer $\mathrm{H}$. Insect-derived chitinases. In: Vilcinskas A, editor. Yellow biotechnology II: Insect biotechnology in plant protection and industry. Berlin, Heidelberg: Springer; 2013. p. 19-50. https://doi.org/10.1007/10_2 013_207.

100. Zhao Y, Li Z, Gu X, Su Y, Liu L. Imaginal disc growth factor 6 (Idgf6) is involved in larval and adult wing development in Bactrocera correcta (Bezzi) (Diptera: Tephritidae). Front Genet. 2020;11:451. https://doi.org/10.3389/ fgene.2020.00451.

101. Uraki R, Hastings AK, Brackney DE, Armstrong PM, Fikrig E. AgBR1 antibodies delay lethal Aedes aegypti-borne West Nile virus infection in mice. NPJ Vaccines. 2019;4(1):23. https://doi.org/10.1038/s41541-019-0120-x.

102. Isaac RE. Neuropeptide-degrading endopeptidase activity of locust (Schistocerca gregaria) synaptic membranes. Biochem J. 1988;255(3):843-7. https://doi.org/10.1042/bj2550843.

103. Gordh G, Legner EF, Caltagirone LE. CHAPTER 15 - Biology of parasitic Hymenoptera. In: Bellows TS, Fisher TW, editors. Handbook of biological control. San Diego: Academic; 1999. p. 355-81. https://doi.org/10.1016/ B978-012257305-7/50062-X.

104. Barrón MG, Fiston-Lavier AS, Petrov DA, González J. Population genomics of transposable elements in Drosophila. Annu Rev Genet. 2014;48(1):561-81. https://doi.org/10.1146/annurev-genet-120213-092359.

105. Burns KH, Boeke JD. Human transposon tectonics. Cell. 2012;149(4):740-52. https://doi.org/10.1016/j.cell.2012.04.019.

106. Feschotte $C$. Transposable elements and the evolution of regulatory networks. Nat Rev Genet. 2008;9(5):397-405. https://doi.org/10.1038/nrg2337.

107. Böhne A, Brunet F, Galiana-Arnoux D, Schultheis C, Volff JN. Transposable elements as drivers of genomic and biological diversity in vertebrates. Chromosome Res. 2008;16(1):203-15. https://doi.org/10.1007/s10577-007-12 02-6.

108. González J, Karasov TL, Messer PW, Petrov DA. Genome-wide patterns of adaptation to temperate environments associated with transposable elements in Drosophila. PLoS Genet. 2010;6(4):e1000905. https://doi.org/1 0.1371/journal.pgen.1000905.

109. Kim YB, Oh JH, Mclver L, Rashkovetsky E, Michalak K, Garner HR, et al. Divergence of Drosophila melanogaster repeatomes in response to a sharp microclimate contrast in Evolution Canyon, Israel. Proc Natl Acad Sci U S A. 2014;111(29):10630-5. https://doi.org/10.1073/pnas.1410372111.

110. Gilbert C, Peccoud J, Cordaux R. Transposable elements and the evolution of insects. Annu Rev Entomol. 2021;66(1):355-72. https://doi.org/10.1146/a nnurev-ento-070720-074650.

111. Dang C, Sun C, Lu Z, Zhong F, Wang F, Wang Q, et al. Cry2A rice did not affect the interspecific interactions between two rice planthoppers, Nilaparvata lugens, and Sogatella furcifera. GM Crops Food. 2019;10(3):17080. https://doi.org/10.1080/21645698.2019.1649530.

112. Chen S, Zhou Y, Chen Y, Gu J. fastp: an ultra-fast all-in-one FASTQ preprocessor. Bioinformatics. 2018;34(17):i884-90. https://doi.org/10.1093/ bioinformatics/bty560.

113. Vurture GW, Sedlazeck FJ, Nattestad M, Underwood CJ, Fang H, Gurtowski J, et al. GenomeScope: fast reference-free genome profiling from short reads. Bioinformatics. 2017;33(14):2202-4. https://doi.org/10.1093/bioinformatics/btx153.

114. Marçais G, Kingsford C. A fast, lock-free approach for efficient parallel counting of occurrences of k-mers. Bioinformatics. 2011;27(6):764-70. https://doi.org/10.1093/bioinformatics/btr011.

115. He K, Lin K, Wang G, Li F. Genome sizes of nine insect species determined by flow cytometry and k-mer analysis. Front Physiol. 2016;7:569.

116. Ruan J, Li H. Fast and accurate long-read assembly with wtdbg2. Nat Methods. 2020;17(2):155-8. https://doi.org/10.1038/s41592-019-0669-3.

117. Hu J, Fan J, Sun Z, Liu S. NextPolish: a fast and efficient genome polishing tool for long-read assembly. Bioinformatics. 2020;36(7):2253-5. https://doi. org/10.1093/bioinformatics/btz891.

118. Seppey M, Manni M, Zdobnov EM. BUSCO: Assessing genome assembly and annotation completeness. Methods Mol Biol Clifton NJ. 1962;2019:227-45.

119. Li H, Durbin R. Fast and accurate short read alignment with BurrowsWheeler transform. Bioinformatics. 2009;25(14):1754-60. https://doi.org/10.1 093/bioinformatics/btp324.

120. Kim D, Paggi JM, Park C, Bennett C, Salzberg SL. Graph-based genome alignment and genotyping with HISAT2 and HISAT-genotype. Nat Biotechnol. 2019;37(8):907-15. https://doi.org/10.1038/s41587-019-0201-4.
121. Li H, Handsaker B, Wysoker A, Fennell T, Ruan J, Homer N, et al. The sequence alignment/map format and SAMtools. Bioinformatics. 2009;25(16): 2078-9. https://doi.org/10.1093/bioinformatics/btp352.

122. Bolger AM, Lohse M, Usadel B. Trimmomatic: a flexible trimmer for Illumina sequence data. Bioinformatics. 2014;30(15):2114-20. https://doi.org/10.1093/ bioinformatics/btu170.

123. Pertea M, Pertea GM, Antonescu CM, Chang TC, Mendell JT, Salzberg SL. StringTie enables improved reconstruction of a transcriptome from RNA-seq reads. Nat Biotechnol. 2015;33(3):290-5. https://doi.org/10.1038/nbt.3122.

124. Li B, Dewey CN. RSEM: accurate transcript quantification from RNA-Seq data with or without a reference genome. BMC Bioinformatics. 2011;12(1):323. https://doi.org/10.1186/1471-2105-12-323.

125. Love Ml, Huber W, Anders S. Moderated estimation of fold change and dispersion for RNA-seq data with DESeq2. Genome Biol. 2014;15(12):550. https://doi.org/10.1186/s13059-014-0550-8.

126. Ou S, Su W, Liao Y, Chougule K, Agda JRA, Hellinga AJ, et al. Benchmarking transposable element annotation methods for creation of a streamlined, comprehensive pipeline. Genome Biol. 2019;20(1):275. https://doi.org/10.11 86/s13059-019-1905-y.

127. Xu Z, Wang H. LTR_FINDER: an efficient tool for the prediction of full-length LTR retrotransposons. Nucleic Acids Res. 2007;35(Suppl 2):W265-8. https:// doi.org/10.1093/nar/gkm286.

128. Ellinghaus D, Kurtz S, Willhoeft U. LTRharvest, an efficient and flexible software for de novo detection of LTR retrotransposons. BMC Bioinformatics. 2008;9(1):18. https://doi.org/10.1186/1471-2105-9-18.

129. Ou S, Jiang N. LTR_retriever: a highly accurate and sensitive program for identification of long terminal repeat retrotransposons. Plant Physiol. 2018; 176(2):1410-22. https://doi.org/10.1104/pp.17.01310.

130. Su W, Gu X, Peterson T. TIR-Learner, a new ensemble method for TIR transposable element annotation, provides evidence for abundant new transposable elements in the maize genome. Mol Plant. 2019;12(3):447-60. https://doi.org/10.1016/j.molp.2019.02.008.

131. Xiong W, He L, Lai J, Dooner HK, Du C. HelitronScanner uncovers a large overlooked cache of Helitron transposons in many plant genomes. Proc Natl Acad Sci U S A. 2014;111(28):10263-8. https://doi.org/10.1073/pnas.1410068111.

132. Flynn JM, Hubley R, Goubert C, Rosen J, Clark AG, Feschotte C, et al. RepeatModeler2 for automated genomic discovery of transposable element families. Proc Natl Acad Sci U S A. 2020;117(17):9451-7. https://doi.org/10.1 073/pnas.1921046117.

133. Tarailo-Graovac M, Chen N. Using RepeatMasker to identify repetitive elements in genomic sequences. Curr Protoc Bioinforma. 2009;25:4.10.1-4.10.14.

134. Bao W, Kojima KK, Kohany O. Repbase Update, a database of repetitive elements in eukaryotic genomes. Mob DNA. 2015;6(1):11. https://doi.org/1 0.1186/s13100-015-0041-9.

135. Benson G. Tandem repeats finder: a program to analyze DNA sequences. Nucleic Acids Res. 1999;27(2):573-80. https://doi.org/10.1093/nar/27.2.573.

136. Haas BJ, Salzberg SL, Zhu W, Pertea M, Allen JE, Orvis J, et al. Automated eukaryotic gene structure annotation using EVidenceModeler and the program to assemble spliced alignments. Genome Biol. 2008;9(1):R7. https:// doi.org/10.1186/gb-2008-9-1-r7.

137. Stanke M, Morgenstern B. AUGUSTUS: a web server for gene prediction in eukaryotes that allows user-defined constraints. Nucleic Acids Res. 2005; 33(Suppl 2):W465-7. https://doi.org/10.1093/nar/gki458.

138. Korf I. Gene finding in novel genomes. BMC Bioinformatics. 2004;5(1):59. https://doi.org/10.1186/1471-2105-5-59.

139. Gremme G, Brendel V, Sparks ME, Kurtz S. Engineering a software tool for gene structure prediction in higher organisms. Inf Softw Technol. 2005; 47(15):965-78. https://doi.org/10.1016/j.infsof.2005.09.005.

140. Conesa A, Götz S, García-Gómez JM, Terol J, Talón M, Robles M. Blast2GO: a universal tool for annotation, visualization and analysis in functional genomics research. Bioinformatics. 2005;21(18):3674-6. https://doi.org/10.1 093/bioinformatics/bti610.

141. Kanehisa M, Sato Y, Morishima K. BlastKOALA and GhostKOALA: KEGG tools for functional characterization of genome and metagenome sequences. J Mol Biol. 2016;428(4):726-31. https://doi.org/10.1016/j.jmb.2015.11.006.

142. Ye XH, Yang Y, Tian Z, Xu L, Yu KL, Xiao S, et al. A high-quality de novo genome assembly from a single parasitoid wasp. 2020. Preprint at https:// www.biorxiv.org/content/10.1101/2020.07.13.200725v1.

143. Rago A, Gilbert DG, Choi J-H, Sackton TB, Wang X, Kelkar YD, et al. OGS2: genome re-annotation of the jewel wasp Nasonia vitripennis. BMC Genomics. 2016;17(1):678. https://doi.org/10.1186/s12864-016-2886-9. 
144. Gatesy J, Baker RH. Hidden likelihood support in genomic data: can fortyfive wrongs make a right? Syst Biol. 2005;54(3):483-92. https://doi.org/10.1 080/10635150590945368.

145. Kubatko LS, Degnan JH. Inconsistency of phylogenetic estimates from concatenated data under coalescence. Syst Biol. 2007;56(1):17-24. https:// doi.org/10.1080/10635150601146041.

146. Zhang C, Rabiee M, Sayyari E, Mirarab S. ASTRAL-III: polynomial time species tree reconstruction from partially resolved gene trees. BMC Bioinformatics. 2018;19(S6):153. https://doi.org/10.1186/s12859-018-2129-y.

147. Yang Z. PAML 4: phylogenetic analysis by maximum likelihood. Mol Biol Evol. 2007;24(8):1586-91. https://doi.org/10.1093/molbev/msm088.

148. Paradis E, Schliep K. ape 5.0: an environment for modern phylogenetics and evolutionary analyses in R. Bioinformatics. 2019;35(3):526-8. https://doi.org/1 0.1093/bioinformatics/bty633.

149. Pond SLK, Frost SDW, Muse SV. HyPhy: hypothesis testing using phylogenies. Bioinformatics. 2005;21(5):676-9. https://doi.org/10.1093/ bioinformatics/bti079.

150. Smith MD, Wertheim JO, Weaver S, Murrell B, Scheffler K, Kosakovsky Pond SL. Less is more: an adaptive branch-site random effects model for efficient detection of episodic diversifying selection. Mol Biol Evol. 2015;32(5):134253. https://doi.org/10.1093/molbev/msv022.

151. Han MV, Thomas GWC, Lugo-Martinez J, Hahn MW. Estimating gene gain and loss rates in the presence of error in genome assembly and annotation using CAFE 3. Mol Biol Evol. 2013;30(8):1987-97. https://doi.org/10.1093/ molbev/mst100

152. Almagro Armenteros JJ, Tsirigos KD, Sønderby CK, Petersen TN, Winther O, Brunak S, et al. SignalP 5.0 improves signal peptide predictions using deep neural networks. Nat Biotechnol. 2019;37(4):420-3. https://doi.org/10.1038/ s41587-019-0036-Z

153. Johnson LS, Eddy SR, Portugaly E. Hidden Markov model speed heuristic and iterative HMM search procedure. BMC Bioinformatics. 2010;11(1):431. https://doi.org/10.1186/1471-2105-11-431.

154. El-Gebali S, Mistry J, Bateman A, Eddy SR, Luciani A, Potter SC, et al. The Pfam protein families database in 2019. Nucleic Acids Res. 2019:47(D1): D427-32. https://doi.org/10.1093/nar/gky995.

155. Zhu Q, Arakane Y, Banerjee D, Beeman RW, Kramer KJ, Muthukrishnan S. Domain organization and phylogenetic analysis of the chitinase-like family of proteins in three species of insects. Insect Biochem Mol Biol. 2008;38(4): 452-66. https://doi.org/10.1016/j.ibmb.2007.06.010.

156. Crawford AM, Brauning R, Smolenski G, Ferguson C, Barton D, Wheeler TI et al. The constituents of Microctonus sp. parasitoid venoms. Insect Mol Biol. 2008;17(3):313-24. https://doi.org/10.1111/j.1365-2583.2008.00802.x.

157. Colinet D, Deleury E, Anselme C, Cazes D, Poulain J, Azema-Dossat C, et al. Extensive inter- and intraspecific venom variation in closely related parasites targeting the same host: the case of Leptopilina parasitoids of Drosophila. Insect Biochem Mol Biol. 2013;43(7):601-11. https://doi.org/10.1016/j.ibmb.2 013.03.010.

158. Dorémus T, Urbach S, Jouan V, Cousserans F, Ravallec M, Demettre E, et al. Venom gland extract is not required for successful parasitism in the polydnavirus-associated endoparasitoid Hyposoter didymator (Hym. Ichneumonidae) despite the presence of numerous novel and conserved venom proteins. Insect Biochem Mol Biol. 2013;43(3):292-307. https://doi. org/10.1016/j.ibmb.2012.12.010.

159. Burke GR, Strand MR. Systematic analysis of a wasp parasitism arsenal. Mol Ecol. 2014:23(4):890-901. https://doi.org/10.1111/mec.12648.

160. Colinet D, Anselme C, Deleury E, Mancini D, Poulain J, Azéma-Dossat C, et al. Identification of the main venom protein components of Aphidius ervi, a parasitoid wasp of the aphid model Acyrthosiphon pisum. BMC Genomics. 2014;15(1):342. https://doi.org/10.1186/1471-2164-15-342.

161. Teng ZW, Xiong SJ, Xu G, Gan SY, Chen X, Stanley D, et al. Protein discovery: Combined transcriptomic and proteomic analyses of venom from the endoparasitoid Cotesia chilonis (Hymenoptera: Braconidae). Toxins. 2017; 9(4):135. https://doi.org/10.3390/toxins9040135.

162. Li LF, Xu ZW, Liu NY, Wu GX, Ren XM, Zhu JY. Parasitism and venom of ectoparasitoid Scleroderma guani impairs host cellular immunity. Arch Insect Biochem Physiol. 2018:98(2):e21451. https://doi.org/10.1002/arch.21451.

163. Tang BZ, Meng E, Zhang HJ, Zhang XM, Asgari S, Lin YP, et al. Combination of label-free quantitative proteomics and transcriptomics reveals intraspecific venom variation between the two strains of Tetrastichus brontispae, a parasitoid of two invasive beetles. J Proteomics. 2019:192:3753. https://doi.org/10.1016/j.jprot.2018.08.003.
164. Alvarado G, Holland SR, DePerez-Rasmussen J, Jarvis BA, Telander T, Wagner $\mathrm{N}$, et al. Bioinformatic analysis suggests potential mechanisms underlying parasitoid venom evolution and function. Genomics. 2020;112(2):1096-104. https://doi.org/10.1016/j.ygeno.2019.06.022.

165. Klopfenstein DV, Zhang L, Pedersen BS, Ramírez F, Warwick Vesztrocy A, Naldi A, et al. GOATOOLS: A Python library for Gene Ontology analyses. Sci Rep. 2018;8(1):10872. https://doi.org/10.1038/s41598-018-28948-z.

166. Yang Y, Ye XH, Dang C, Cao YS, Hong, R, Sun HY, et al. Gonatopus flavifemur, genome sequencing and assembly. BioProject. 2021. https:// identifiers.org/ncbi/bioproject:PRJNA695321.

167. Yang Y. Gonatopus flavifemur isolate zju, whole genome shotgun sequencing project. 2021. GenBank https://identifiers.org/ncbi/insdc:JA FFJZO00000000

168. Yang Y, Ye XH, Dang C, Cao YS, Hong R, Sun Y, et al. Gonatopus flavifemur, genome sequencing and assembly. 2021. InsectBase http://insect-genome. com/Gfla.

\section{Publisher's Note}

Springer Nature remains neutral with regard to jurisdictional claims in published maps and institutional affiliations.
Ready to submit your research? Choose BMC and benefit from:

- fast, convenient online submission

- thorough peer review by experienced researchers in your field

- rapid publication on acceptance

- support for research data, including large and complex data types

- gold Open Access which fosters wider collaboration and increased citations

- maximum visibility for your research: over $100 \mathrm{M}$ website views per year

At BMC, research is always in progress.

Learn more biomedcentral.com/submissions 